Interfaces and Free Boundaries 13 (2011), 127-154

DOI $10.4171 / \mathrm{IFB} / 251$

\title{
A toroidal tube solution to a problem involving mean curvature and Newtonian potential
}

\author{
XIAOFENG REN \\ Department of Mathematics, The George Washington University, Washington, DC 20052, USA \\ E-mail: ren@gwu.edu \\ JUNCHENG WEI \\ Department of Mathematics, The Chinese University of Hong Kong, Hong Kong, PRC \\ E-mail:wei@math.cuhk.edu.hk
}

[Received 11 November 2009 and in revised form 10 June 2010]

\begin{abstract}
The Ohta-Kawasaki theory for block copolymer morphology and the Gierer-Meinhardt theory for morphogenesis in cell development both give rise to a nonlocal geometric problem. One seeks a set in $\mathbb{R}^{3}$ which satisfies an equation that links the mean curvature of the boundary of the set to the Newtonian potential of the set. An axisymmetric, torus shaped, tube like solution exists in $\mathbb{R}^{3}$ if the lone parameter of the problem is sufficiently large. A cross section of the torus is small and the distance from the center of the cross section to the axis of symmetry is large. The solution is stable in the class of axisymmetric sets in a particular sense. This work is motivated by the recent discovery of a toroidal morphological phase in a triblock copolymer.
\end{abstract}

2010 Mathematics Subject Classification: 35R35, 82B24, 82D60.

Keywords: Torus; tube; axisymmetry; nonlocal geometric problem; mean curvature; Newtonian potential; approximate solution; Lyapunov-Schmidt reduction.

\section{Introduction}

Ring shaped objects in space have been observed in many physical systems. Known as the vortex ring in fluid dynamics, it is a region of rotating fluid where the flow pattern takes on a toroidal shape [3]. In a quantum fluid, a vortex ring is formed by a loop of poloidal quantized flow pattern. It was detected in superfluid helium by Rayfield and Reif [27], and more recently in Bose-Einstein condensates by Anderson et al. [2].

Our study is inspired by recent experimental findings in the block copolymer research. Block copolymers are soft condensed materials characterized by fluid-like disorder on the molecular scale and a high degree of order on a longer length scale. Various morphological phases have been observed as orderly outcomes of the microphase separation property [4]. In 2004, Pochan et al. produced a morphological phase of toroidal supramolecule assemblies, using a triblock copolymer [26].

In this paper we show the existence of a torus shaped set as a solution of a nonlocal geometric problem: Given two parameters $m, \gamma>0$, find a set $E$ in $\mathbb{R}^{3}$ and a number $\lambda$ such that the volume of $E$ is $m$ and on the boundary of $E$ the equation

$$
\mathcal{H}(\partial E)+\gamma \mathcal{N}(E)=\lambda
$$


holds. In 1.1$), \mathcal{H}(\partial E)$ stands for the mean curvature of the boundary of $E$, and $\mathcal{N}$ is the Newtonian potential operator

$$
\mathcal{N}(E)(x)=\int_{E} \frac{1}{4 \pi|x-y|} \mathrm{d} y .
$$

The unknown constant $\lambda$ is the Lagrange multiplier associated with the constraint that the volume of $E$ is $m$. If the problem is considered in $\mathbb{R}^{2}$, then $\mathcal{H}(\partial E)$ is the curvature of the curve $\partial E$ and $\mathcal{N}$ takes the form

$$
\mathcal{N}(E)(x)=\int_{E} \frac{1}{2 \pi} \log \frac{1}{|x-y|} \mathrm{d} y .
$$

The equation (1.1) admits a variational structure. It is the Euler-Lagrange equation of the functional

$$
\mathcal{J}(E)=\frac{1}{2} \mathcal{P}(E)+\frac{\gamma}{2} \int_{E} \mathcal{N}(E)(x) \mathrm{d} x .
$$

Here $\mathcal{P}(E)$ stands for the perimeter of $E$, i.e. the area of $\partial E$.

There is an analogy to 1.1 on bounded domains. Let $D \subset \mathbb{R}^{3}$ be a bounded and smooth domain and $a \in(0,1)$ and $\gamma>0$ be two parameters. We look for a subset $E$ of $D$ whose volume is $a$ times the volume of $D$, and a number $\lambda$ such that the equation

$$
\mathcal{H}\left(\partial_{D} E\right)+\gamma(-\Delta)^{-1}\left(\chi_{E}-a\right)=\lambda
$$

holds on $\partial_{D} E$, the part of the boundary of $E$ that is in $D$. If $\partial E$ meets $\partial D$, we require that they meet orthogonally. Here $(-\Delta)^{-1}$ is the inverse of $-\Delta$ and $-\Delta$ acts on functions with the zero Neumann boundary condition on $\partial D$.

The problem (1.3) may be deduced from the Ohta-Kawasaki theory for block copolymers [24] as a strong segregation limit. This connection was first observed by Nishiura and Ohnishi [23]. In [29] this limit was shown to be a $\Gamma$-limit [6, 21, 20, 16]; see the appendix for more details.

This problem is also connected to the Gierer-Meinhardt system with saturation. The GiererMeinhardt system is an activator-inhibitor type reaction diffusion PDE system. It was first introduced to model the head formation of hydra [13]. More generally it can be used to study morphogenesis in cell development [18, 19, 22]. It is a minimal model that provides a theoretical bridge between observations on the one hand and the deduction of the underlying molecular-genetic mechanisms on the other hand. More information on the Gierer-Meinhardt type PDE systems may be found in [11, 12].

In [7] del Pino discovered a radially symmetric solution to the Gierer-Meinhardt system whose $u$ component has one transition layer. The parameter range of the Gierer-Meinhardt system he considered does not quite lead to (1.3). Later Sakamoto and Suzuki found a similar radially symmetric solution with one transition layer in a slightly different parameter range [36]. The $u$ component of their solution tends to a constant multiple of the characteristic function of a ball as $\epsilon \rightarrow 0$. Their parameter turns out to be directly related to (1.3), and their limiting ball is a solution to 1.3 .

Due to the lack of a variational structure, the connection between the Gierer-Meinhardt system in the parameter range considered in [36] and the nonlocal geometric problem (1.3) cannot be interpreted as a $\Gamma$-convergence property. However an asymptotic analysis can be employed to show formally that the activator of a steady state of the system is close to the characteristic function of a set $E$, which satisfies the same equation (1.3); see the appendix. 
The equation (1.3) was solved completely in one dimension where the domain is a bounded interval [29]. There are countably infinitely many solutions and every solution is a local minimizer of the energy functional associated with (1.3). Among these local minimizers, two or four are global minimizers. In higher dimensions many types of solutions to $(1.3)$ have been found [30-35, 25, 14 , 28, 15, 5]. The global minimizer has not been completely identified [1, 37]. There are several ways to associate dynamic models to the stationary problem (1.3). One case was studied in [10] where every one-dimensional initial state converges to one of the local minimizers found in [29].

There is a strong connection between (1.1) and (1.3). One may use solutions to 1.1 as building blocks to construct solutions to 1.3 . For instance, in $\mathbb{R}^{2}$ the unit disc is a solution of (1.1). This is because the boundary of the disc, the unit circle, has constant curvature, and the Newtonian potential of the unit disc is a radially symmetric function and therefore a constant on the boundary of the disc. The sum of these two constants is again a constant and hence (1.1) is satisfied by the unit disc. Using this unit disc we found solutions to (1.3) with multiple components, each of which is close to a small disc (a scaled-down version of the unit disc) [32]. When the number of components is large, they form a hexagonal pattern. This gives a mathematical explanation for the cylindrical morphology phase in diblock copolymers. More discussion on the relationship between (1.1) and 1.3 is given in the last section.

In this paper we construct a solution which has the shape of a toroidal tube as depicted in Figure 1. It is a set obtained by rotating a small, approximately round disc in the $y z$-plane, far from the $z$-axis, about the $z$-axis.

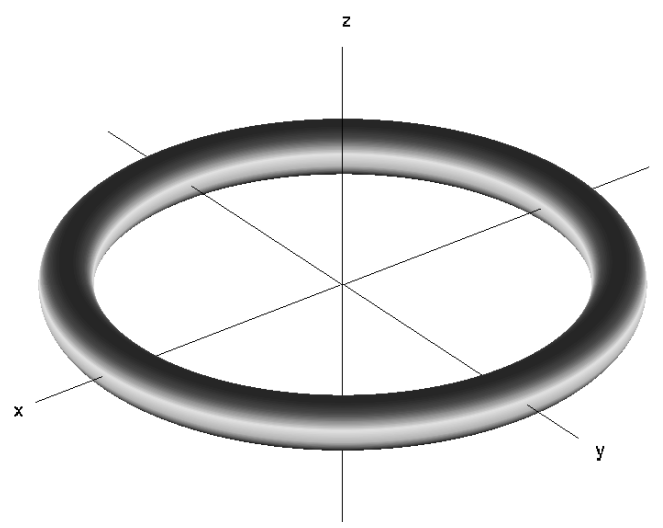

FIG. 1. A toroidal tube like solution.

The two parameters $m$ and $\gamma$ can be reduced to one. Without loss of generality we assume that

$$
m=1 .
$$

The case $m \neq 1$ can be reduced to the case $m=1$ by a change of space variable and a change of $\gamma$ accordingly. Therefore the problem (1.1) has intrinsically only one parameter, which we take to be $\gamma$.

We define a function $f=f(\gamma)$ from $(0, \infty)$ to $(0, \infty)$ through its inverse

$$
\gamma=\frac{2}{f^{3} \log \frac{1}{2 \pi^{2} f^{3}}} .
$$

Note that $f(\gamma) \rightarrow 0$ as $\gamma \rightarrow \infty$. Our main result is the following existence theorem. 
THEOREM 1.1 When $\gamma$ is large enough, (1.1) admits a solution of unit volume which is close to a set enclosed by a torus. The torus is obtained by rotating a circle in the $y z$-plane about the $z$-axis. Let $p_{\gamma}$ be the distance from the center of the circle to the $z$-axis and $q_{\gamma}$ be the radius of the circle. Then

$$
2 \pi^{2} p_{\gamma} q_{\gamma}^{2}=1, \quad \lim _{\gamma \rightarrow \infty} \frac{q_{\gamma}}{f(\gamma)}=1, \quad \lim _{\gamma \rightarrow \infty} 2 \pi^{2} f^{2}(\gamma) p_{\gamma}=1 .
$$

In the proof of the theorem we will see in what sense the solution is close to the region enclosed by the torus.

The theorem is proved by mathematically rigorous singular perturbation techniques developed in [33, 32, 34] for this type of nonlocal geometric problems. The proof consists of several steps. In Section 2 we represent the Newtonian potential in (1.1) by an integral operator whose kernel is a Green's function in the class of axisymmetric functions. The type of the singularity of this Green's function is determined in Lemma 2.1. In Section 3 a family of approximate solutions, sets bounded by tori, are studied. Lemma 3.2 shows how much error an approximate solution generates when plugged into (1.1). The value of $\mathcal{J}$ at an approximate solution is estimated in Lemma 3.3 . In Section 4 we specify a way to perturb each approximate solution and recast the problem (1.1) in terms of the perturbation. Lemma 4.1 contains the properties of the linearized operator at each approximate solution. In Section 5 we carry out a Lyapunov-Schmidt type argument to find a particular perturbation to each approximate solution so that the exact solution of (1.1) is in the family of the perturbed approximate solutions. In the last section we identify the exact solution in this family. The connections between the Ohta-Kawasaki theory, the Gierer-Meinhardt system, and (1.3) are explained in the appendix.

In this paper $C$ denotes a constant independent of the parameters in the construction process. Its value may vary from place to place. Functions that are $2 \pi$-periodic are considered to be defined on the unit circle $S^{1}$. For example $H^{2}\left(S^{1}\right)$ denotes the $W^{2,2}$ Sobolev space of $2 \pi$-periodic functions.

\section{Green's function}

Axisymmetric objects are best described by cylindrical coordinates, i.e. $x=r \cos \sigma, y=r \sin \sigma$, and $z=z$, so that they are independent of the angle variable $\sigma$. The variables $r$ and $z$ are in

$$
\mathbb{R}_{+}^{2}=\{(r, z): r>0, z \in \mathbb{R}\}
$$

An axisymmetric object $E$ in $\mathbb{R}^{3}$ is identified by its description in $\mathbb{R}_{+}^{2}$, so for simplicity we view $E$ as a subset of $\mathbb{R}_{+}^{2}$. On such a set the $\mathcal{N}$ operator takes the form

$$
\mathcal{N}(E)(r, z)=\int_{E} G(r, z, s, t) \mathrm{d} s \mathrm{~d} t
$$

where $E \subset \mathbb{R}_{+}^{2}$ and the function $G$ is our Green's function. By $(1.2), G$ may be represented by

$$
G(r, z, s, t)=\frac{s}{4 \pi} \int_{0}^{2 \pi} \frac{\mathrm{d} \sigma}{\sqrt{r^{2}+s^{2}-2 r s \cos \sigma+(z-t)^{2}}} .
$$

Moreover for each $(s, t) \in \mathbb{R}_{+}^{2}$ as a function of $(r, z), G(\cdot, s, t)$ satisfies

$$
-\Delta G(\cdot, s, t)-\frac{\partial}{r \partial r} G(\cdot, s, t)=\delta_{(s, t)} \quad \text { in } \mathbb{R}_{+}^{2}, \quad \frac{\partial}{\partial r} G(0, z, s, t)=0 \quad \forall z \in \mathbb{R} .
$$


LEMmA 2.1 For each $(s, t) \in \mathbb{R}_{+}^{2}$,

$$
\begin{aligned}
& G(r, z, s, t) \\
& =\frac{1}{2 \pi} \log \frac{1}{|(r, z)-(s, t)|}+G_{1}(r, z, s, t) \\
& =\frac{1}{2 \pi} \log \frac{1}{|(r, z)-(s, t)|}-\frac{r-s}{4 \pi s} \log \frac{1}{|(r, z)-(s, t)|}+G_{2}(r, z, s, t) \\
& =\frac{1}{2 \pi} \log \frac{1}{|(r, z)-(s, t)|}-\frac{r-s}{4 \pi s} \log \frac{1}{|(r, z)-(s, t)|}+\frac{5(r-s)^{2}-(z-t)^{2}}{32 \pi s^{2}} \log \frac{1}{|(r, z)-(s, t)|} \\
& \quad+G_{3}(r, z, s, t)
\end{aligned}
$$

where, as functions of $(r, z), G_{1}(\cdot, s, t) \in C_{\text {loc }}^{\alpha}\left(\mathbb{R}_{+}^{2}\right), G_{2}(\cdot, s, t) \in C_{\text {loc }}^{1, \alpha}\left(\mathbb{R}_{+}^{2}\right)$, and $G_{3}(\cdot, s, t) \in$ $C_{\text {loc }}^{2, \alpha}\left(\mathbb{R}_{+}^{2}\right)$ for all $\alpha \in(0,1)$.

Proof. Since

$$
-\frac{r-s}{4 \pi s} \log \frac{1}{|(r, z)-(s, t)|} \in C_{\mathrm{loc}}^{\alpha}\left(\mathbb{R}_{+}^{2}\right) \quad \text { and } \quad \frac{5(r-s)^{2}-(z-t)^{2}}{32 \pi s^{2}} \log \frac{1}{|(r, z)-(s, t)|} \in C_{\mathrm{loc}}^{1, \alpha}\left(\mathbb{R}_{+}^{2}\right),
$$

it suffices to show that $G_{3}(\cdot, s, t) \in C_{\text {loc }}^{2, \alpha}\left(\mathbb{R}_{+}^{2}\right)$. Compute

$$
\begin{aligned}
\left(-\Delta-\frac{\partial}{r \partial r}\right)\left[\frac{1}{2 \pi} \log \right. & \frac{1}{|(r, z)-(s, t)|}-\frac{r-s}{4 \pi s} \log \frac{1}{|(r, z)-(s, t)|} \\
= & -\Delta\left(\frac{1}{2 \pi} \log \frac{1}{|(r, z)-(s, t)|}\right)-\frac{\partial}{r \partial r}\left(\frac{1}{2 \pi} \log \frac{1}{|(r, z)-(s, t)|}\right) \\
& +\Delta\left(\frac{r-s}{4 \pi s} \log \frac{1}{|(r, z)-(s, t)|}\right)+\frac{\partial}{r \partial r}\left(\frac{r-s}{4 \pi s} \log \frac{1}{|(r, z)-(s, t)|}\right) \\
& -\Delta\left(\frac{5(r-s)^{2}-(z-t)^{2}}{32 \pi s^{2}} \log \frac{1}{|(r, z)-(s, t)|}\right) \\
& -\frac{\partial}{r \partial r}\left(\frac{5(r-s)^{2}-(z-t)^{2}}{32 \pi s^{2}} \log \frac{1}{|(r, z)-(s, t)|}\right) \\
= & \delta(s, t)+\frac{r-s}{2 \pi r|(r, z)-(s, t)|^{2}} \\
& -\frac{r-s}{2 \pi s|(r, z)-(s, t)|^{2}}+\frac{1}{4 \pi r s} \log \frac{1}{|(r, z)-(s, t)|}-\frac{(r-s)^{2}}{4 \pi r s|(r, z)-(s, t)|^{2}} \\
& -\frac{1}{4 \pi s^{2}} \log \frac{1}{|(r, z)-(s, t)|}+\frac{5(r-s)^{2}-(z-t)^{2}}{8 \pi s^{2}|(r, z)-(s, t)|^{2}} \\
& -\frac{5(r-s)}{16 \pi r s^{2}} \log \frac{1}{|(r, z)-(s, t)|}+\frac{5(r-s)^{3}-(z-t)^{2}(r-s)}{32 \pi r s^{2}|(r, z)-(s, t)|^{2}} . \\
& \\
& \\
& \\
&
\end{aligned}
$$


Note that the above is $\delta_{(s, t)}$ plus a $C_{\text {loc }}^{\alpha}\left(\mathbb{R}_{+}^{2}\right)$ function, since

$$
\begin{array}{r}
\frac{r-s}{2 \pi r|(r, z)-(s, t)|^{2}}-\frac{r-s}{2 \pi s|(r, z)-(s, t)|^{2}}-\frac{(r-s)^{2}}{4 \pi r s|(r, z)-(s, t)|^{2}}+\frac{5(r-s)^{2}-(z-t)^{2}}{8 \pi s^{2}|(r, z)-(s, t)|^{2}} \\
\frac{1}{4 \pi r s} \log \frac{1}{|(r, z)-(s, t)|}-\frac{1}{4 \pi s^{2}} \log \frac{1}{|(r, z)-(s, t)|} \in C_{\mathrm{loc}}^{\alpha}\left(\mathbb{R}_{+}^{2}\right), \\
-\frac{5(r-s)}{16 \pi r s^{2}} \log \frac{1}{|(r, z)-(s, t)|} \in C_{\mathrm{loc}}^{\alpha}\left(\mathbb{R}_{+}^{2}\right), \\
\frac{5(r-s)^{3}-(z-t)^{2}(r-s)}{32 \pi r s^{2}|(r, z)-(s, t)|^{2}} \in C_{\mathrm{loc}}^{0,1}\left(\mathbb{R}_{+}^{2}\right) .
\end{array}
$$

Here $C_{\text {loc }}^{0,1}\left(\mathbb{R}_{+}^{2}\right)$ denotes the space of locally Lipschitz continuous functions on $\mathbb{R}_{+}^{2}$. Because of 2.3 , we deduce

$$
\left(-\Delta-\frac{\partial}{r \partial r}\right) G_{3}(\cdot, s, t) \in C_{\mathrm{loc}}^{\alpha}\left(\mathbb{R}_{+}^{2}\right) .
$$

The elliptic regularity theory implies that $G_{3}(\cdot, s, t) \in C_{\text {loc }}^{2, \alpha}\left(\mathbb{R}_{+}^{2}\right)$.

Some useful properties of $G, G_{1}, G_{2}$ and $G_{3}$ are listed below.

Lemma 2.2 Denote the derivatives of $G=G(r, z, s, t)$ ( $\left(\right.$ r $G_{1}, G_{2}, G_{3}$ ) with respect to $r, z, s$, and $t$ by $D_{1} G, D_{2} G, D_{3} G$ and $D_{4} G$ respectively.

(i) For $\lambda>0, G(\lambda r, \lambda z, \lambda s, \lambda t)=G(r, z, s, t)$.

(ii) For $r \neq s, D_{2} G(r, 0, s, 0)=0$.

(iii) For $r>0$ and $s>0, D_{2} G_{3}(r, 0, s, 0)=0$.

Proof. Parts (i) and (ii) follow directly from (2.2). To prove (iii), we first assume $r \neq s$ and deduce from $D_{2} G(r, 0, s, 0)=0$ in (ii) that

$$
\begin{aligned}
D_{2} G_{3}(r, 0, s, 0)= & -D_{2}\left[\frac{1}{2 \pi} \log \frac{1}{|(r, z)-(s, t)|}-\frac{r-s}{4 \pi s} \log \frac{1}{|(r, z)-(s, t)|}\right. \\
& \left.+\frac{5(r-s)^{2}-(z-t)^{2}}{32 \pi s^{2}} \log \frac{1}{|(r, z)-(s, t)|}\right]\left.\right|_{z=t=0} \\
= & -\left[-\frac{z-t}{2 \pi|(r, z)-(s, t)|^{2}}+\frac{(r-s)(z-t)}{4 \pi s|(r, z)-(s, t)|^{2}}\right. \\
& \left.-\frac{z-t}{16 \pi s^{2}} \log \frac{1}{|(r, z)-(s, t)|}-\frac{5(r-s)^{2}(z-t)-(z-t)^{3}}{32 \pi s^{2}|(r, z)-(s, t)|^{2}}\right]_{z=t=0} \\
= & 0 .
\end{aligned}
$$

Since $G_{3}(\cdot, s, t) \in C_{\mathrm{loc}}^{2, \alpha}\left(\mathbb{R}_{+}^{2}\right), D_{2} G_{3}(r, 0, s, 0)=0$ remains valid even if $r=s$.

\section{Approximate solutions}

A perfect torus is obtained by rotating a circle of radius $q$ about the $z$-axis. Due to the translation invariance of this problem in the $z$-direction, without loss of generality the center of the circle is assumed to be in the $x y$-plane. Suppose that the distance of the center of the circle to the $z$-axis is $p$ where $p>q>0$. The region inside the torus is denoted by $E_{0}$ whose volume is $2 \pi^{2} p q^{2}$, and 
because of the constraint (1.4) we have

$$
2 \pi^{2} p q^{2}=1
$$

The region $E_{0}$ is regarded as an approximate solution, parametrized by $p$. The next lemma estimates the effect of $\mathcal{N}$ on $E_{0}$.

LEMMA $3.1 \mathcal{N}\left(E_{0}\right)$ on $E_{0}$ is

$\mathcal{N}\left(E_{0}\right)(p+q \hat{h} \cos \theta, q \hat{h} \sin \theta)=\frac{q^{2}}{2} \log \frac{1}{q}+q^{2}\left[\pi G_{3}(p, 0, p, 0)+\frac{1}{4}-\frac{\hat{h}^{2}}{4}\right]$

where $\hat{h} \in[0,1]$.

$$
-\frac{q^{3}}{4 p}\left(\log \frac{1}{q}\right) \hat{h} \cos \theta+\frac{q^{3}}{p}\left[\pi p D_{1} G_{3}(p, 0, p, 0) \hat{h}+\frac{\hat{h}^{3}}{16}\right] \cos \theta+O\left(\frac{q^{4}}{p^{2}} \log \frac{p}{q}\right),
$$

Proof. In the Green's function $G(r, z, s, t)$ let

$$
(r, z)=(p+h \cos \theta, h \sin \theta), \quad(s, t)=(p+\rho \cos \omega, \rho \sin \omega)
$$

where $h, \rho \in[0, q]$, so both $(r, z)$ and $(s, t)$ are points in $E_{0}$. Then by Lemma 2.1 .

$$
\begin{aligned}
\mathcal{N}\left(E_{0}\right)(p+h & \cos \theta, h \sin \theta) \\
= & \int_{0}^{q} \int_{0}^{2 \pi} G(p+h \cos \theta, h \sin \theta, p+\rho \cos \omega, \rho \sin \omega) \rho \mathrm{d} \omega \mathrm{d} \rho \\
= & \int_{0}^{q} \int_{0}^{2 \pi} \frac{1}{2 \pi} \log \frac{1}{\sqrt{h^{2}+\rho^{2}-2 h \rho \cos (\theta-\omega)}}\left[1-\frac{h \cos \theta-\rho \cos \omega}{2(p+\rho \cos \omega)}\right. \\
& \left.+\frac{5(h \cos \theta-\rho \cos \omega)^{2}-(h \sin \theta-\rho \sin \omega)^{2}}{16(p+\rho \cos \omega)^{2}}\right] \rho \mathrm{d} \omega \mathrm{d} \rho \\
& +\int_{0}^{q} \int_{0}^{2 \pi} G_{3}(p+h \cos \theta, h \sin \theta, p+\rho \cos \omega, \rho \sin \omega) \rho \mathrm{d} \omega \mathrm{d} \rho \\
= & I+I I
\end{aligned}
$$

where $I$ and $I I$ in 3.2 are given by the two integrals above.

Because of the scaling property

$$
G_{3}(\lambda r, \lambda z, \lambda s, \lambda t)=G_{3}(r, z, s, t)-\frac{1}{2 \pi} \log \frac{1}{\lambda}\left[1-\frac{r-s}{2 s}+\frac{5(r-s)^{2}-(z-t)^{2}}{16 s^{2}}\right],
$$

which follows from Lemmas 2.2 (i) and 2.1, II becomes

$$
\begin{aligned}
I I= & -\frac{1}{2 \pi} \log \frac{1}{p} \int_{0}^{q} \int_{0}^{2 \pi}\left[1-\frac{h \cos \theta-\rho \cos \omega}{2(p+\rho \cos \omega)}\right. \\
& \left.+\frac{5(h \cos \theta-\rho \cos \omega)^{2}-(h \sin \theta-\rho \sin \omega)^{2}}{16(p+\rho \cos \omega)^{2}}\right] \rho \mathrm{d} \omega \mathrm{d} \rho \\
& +\int_{0}^{q} \int_{0}^{2 \pi} G_{3}\left(1+\frac{h}{p} \cos \theta, \frac{h}{p} \sin \theta, 1+\frac{\rho}{p} \cos \omega, \frac{\rho}{p} \sin \omega\right) \rho \mathrm{d} \omega \mathrm{d} \rho \\
= & I_{I}+I_{I I}
\end{aligned}
$$

where $I_{I}$ and $I I_{I I}$ are given by the two summands above. 
We introduce the rescaled variables $\hat{h}$ and $\hat{\rho}$ by

$$
h=q \hat{h}, \quad \rho=q \hat{\rho} .
$$

Then $I$ becomes

$$
\begin{aligned}
I= & \frac{q^{2}}{2 \pi} \log \frac{1}{q} \int_{0}^{1} \int_{0}^{2 \pi}\left[1-\frac{\hat{h} \cos \theta-\hat{\rho} \cos \omega}{2(p / q+\hat{\rho} \cos \omega)}\right. \\
& \left.+\frac{5(\hat{h} \cos \theta-\hat{\rho} \cos \omega)^{2}-(\hat{h} \sin \theta-\hat{\rho} \sin \omega)^{2}}{16(p / q+\hat{\rho} \cos \omega)^{2}}\right] \hat{\rho} \mathrm{d} \omega \mathrm{d} \hat{\rho} \\
& +\frac{q^{2}}{2 \pi} \int_{0}^{1} \int_{0}^{2 \pi} \log \frac{1}{\sqrt{\hat{h}^{2}+\hat{\rho}^{2}-2 \hat{h} \hat{\rho} \cos (\theta-\omega)}}\left[1-\frac{\hat{h} \cos \theta-\hat{\rho} \cos \omega}{2(p / q+\hat{\rho} \cos \omega)}\right. \\
& \left.+\frac{5(\hat{h} \cos \theta-\hat{\rho} \cos \omega)^{2}-(\hat{h} \sin \theta-\hat{\rho} \sin \omega)^{2}}{16(p / q+\hat{\rho} \cos \omega)^{2}}\right] \hat{\rho} \mathrm{d} \omega \mathrm{d} \hat{\rho} \\
= & I_{I}+I_{I I}
\end{aligned}
$$

where $I_{I}$ and $I_{I I}$ are the two summands above.

Combining $I_{I}$ and $I_{I}$ we find

$$
\begin{aligned}
I_{I}+I_{I}= & \frac{q^{2}}{2 \pi} \log \frac{p}{q} \int_{0}^{1} \int_{0}^{2 \pi}\left[1-\frac{\hat{h} \cos \theta-\hat{\rho} \cos \omega}{2(p / q+\hat{\rho} \cos \omega)}\right. \\
& \left.+\frac{5(\hat{h} \cos \theta-\hat{\rho} \cos \omega)^{2}-(\hat{h} \sin \theta-\hat{\rho} \sin \omega)^{2}}{16(p / q+\hat{\rho} \cos \omega)^{2}}\right] \hat{\rho} \mathrm{d} \omega \mathrm{d} \hat{\rho} \\
= & \frac{q^{2}}{2 \pi} \log \frac{p}{q}[A+B+C]
\end{aligned}
$$

where

$$
\begin{aligned}
A & =\int_{0}^{1} \int_{0}^{2 \pi} \hat{\rho} \mathrm{d} \omega \mathrm{d} \hat{\rho}=\pi, \\
B & =\int_{0}^{1} \int_{0}^{2 \pi}-\frac{\hat{h} \cos \theta-\hat{\rho} \cos \omega}{2(p / q+\hat{\rho} \cos \omega)} \hat{\rho} \mathrm{d} \omega \mathrm{d} \hat{\rho} \\
& =-\frac{q}{2 p} \int_{0}^{1} \int_{0}^{2 \pi}\left(\hat{h} \cos \theta-\hat{\rho} \cos \omega+O\left(\frac{q}{p}\right)\right) \hat{\rho} \mathrm{d} \omega \mathrm{d} \hat{\rho}=-\frac{\pi q}{2 p} \hat{h} \cos \theta+O\left(\frac{q^{2}}{p^{2}}\right) \\
C & =\int_{0}^{1} \int_{0}^{2 \pi} \frac{5(\hat{h} \cos \theta-\hat{\rho} \cos \omega)^{2}-(\hat{h} \sin \theta-\hat{\rho} \sin \omega)^{2}}{16(p / q+\hat{\rho} \cos \omega)^{2}} \hat{\mathrm{d} \omega} \mathrm{d} \hat{\rho}=O\left(\frac{q^{2}}{p^{2}}\right) .
\end{aligned}
$$

This shows

$$
I_{I}+I_{I}=\frac{q^{2}}{2} \log \frac{p}{q}-\frac{q^{3}}{4 p}\left(\log \frac{p}{q}\right) \hat{h} \cos \theta+O\left(\frac{q^{4}}{p^{2}} \log \frac{p}{q}\right)
$$


The term $I_{I I}$ must be estimated carefully. We write

$$
\begin{aligned}
I_{I I}= & \frac{q^{2}}{2 \pi} \int_{0}^{1} \int_{0}^{2 \pi} \log \frac{1}{\sqrt{\hat{h}^{2}+\hat{\rho}^{2}-2 \hat{h} \hat{\rho} \cos (\theta-\omega)}}\left[1-\frac{\hat{h} \cos \theta-\hat{\rho} \cos \omega}{2(p / q+\hat{\rho} \cos \omega)}\right. \\
& \left.+\frac{5(\hat{h} \cos \theta-\hat{\rho} \cos \omega)^{2}-(\hat{h} \sin \theta-\hat{\rho} \sin \omega)^{2}}{16(p / q+\hat{\rho} \cos \omega)^{2}}\right] \hat{\rho} \mathrm{d} \omega \mathrm{d} \hat{\rho} \\
= & \frac{q^{2}}{2 \pi}[\tilde{A}+\tilde{B}+\tilde{C}]
\end{aligned}
$$

where

$$
\begin{aligned}
\tilde{A}= & \int_{0}^{1} \int_{0}^{2 \pi} \log \frac{1}{\sqrt{\hat{h}^{2}+\hat{\rho}^{2}-2 \hat{h} \hat{\rho} \cos (\theta-\omega)}} \hat{\rho} \mathrm{d} \omega \mathrm{d} \hat{\rho}, \\
\tilde{B}= & -\int_{0}^{1} \int_{0}^{2 \pi} \log \frac{1}{\sqrt{\hat{h}^{2}+\hat{\rho}^{2}-2 \hat{h} \hat{\rho} \cos (\theta-\omega)}} \frac{\hat{h} \cos \theta-\hat{\rho} \cos \omega}{2(p / q+\hat{\rho} \cos \omega)} \hat{\rho} \mathrm{d} \omega \mathrm{d} \hat{\rho}, \\
\tilde{C}= & \int_{0}^{1} \int_{0}^{2 \pi} \log \frac{1}{\sqrt{\hat{h}^{2}+\hat{\rho}^{2}-2 \hat{h} \hat{\rho} \cos (\theta-\omega)}} \\
& \times \frac{5(\hat{h} \cos \theta-\hat{\rho} \cos \omega)^{2}-(\hat{h} \sin \theta-\hat{\rho} \sin \omega)^{2}}{16(p / q+\hat{\rho} \cos \omega)^{2}} \hat{\rho} \mathrm{d} \omega \mathrm{d} \hat{\rho}=O\left(\frac{q^{2}}{p^{2}}\right) .
\end{aligned}
$$

To study $\tilde{A}$ and $\tilde{B}$ we need the trigonometric series

$$
\log \frac{1}{\mid 1-\beta e^{i \omega \mid}}=\sum_{n=1}^{\infty} \frac{\beta^{n} \cos n \omega}{n}
$$

for $\beta \in[0,1]$. One consequence of $[3.6]$ is that

$$
\int_{0}^{2 \pi} \log \frac{1}{\sqrt{\hat{h}^{2}+\hat{\rho}^{2}-2 \hat{h} \hat{\rho} \cos (\theta-\omega)}} \mathrm{d} \omega= \begin{cases}-2 \pi \log \hat{h} & \text { if } \hat{\rho}<\hat{h}, \\ -2 \pi \log \hat{\rho} & \text { if } \hat{\rho} \geqslant \hat{h},\end{cases}
$$

which implies that

$$
\tilde{A}=-2 \pi \int_{0}^{\hat{h}}(\log \tilde{h}) \hat{\rho} \mathrm{d} \hat{\rho}-2 \pi \int_{\hat{h}}^{1}(\log \tilde{\rho}) \hat{\rho} \mathrm{d} \hat{\rho}=2 \pi\left(\frac{1}{4}-\frac{\hat{h}^{2}}{4}\right)
$$

Regarding $\tilde{B}$ we write

$$
\begin{aligned}
\tilde{B}= & -\frac{q}{2 p} \int_{0}^{1} \int_{0}^{2 \pi} \log \frac{1}{\sqrt{\hat{h}^{2}+\hat{\rho}^{2}-2 \hat{h} \hat{\rho} \cos (\theta-\omega)}} \hat{h} \cos \theta \hat{\rho} \mathrm{d} \omega \mathrm{d} \hat{\rho} \\
& +\frac{q}{2 p} \int_{0}^{1} \int_{0}^{2 \pi} \log \frac{1}{\sqrt{\hat{h}^{2}+\hat{\rho}^{2}-2 \hat{h} \hat{\rho} \cos (\theta-\omega)}} \hat{\rho} \cos \omega \hat{\rho} \mathrm{d} \omega \mathrm{d} \hat{\rho}+O\left(\frac{q^{2}}{p^{2}}\right) \\
= & \tilde{B}_{1}+\tilde{B}_{2}+O\left(\frac{q^{2}}{p^{2}}\right)
\end{aligned}
$$

where $\tilde{B}_{1}$ and $\tilde{B}_{2}$ are the first two summands. 
Using (3.7) again, we derive

$$
\begin{aligned}
\tilde{B}_{1} & =-\frac{q}{2 p}\left[\int_{0}^{\hat{h}}(-2 \pi \log \hat{h}) \hat{h} \cos \theta \hat{\rho} \mathrm{d} \hat{\rho}+\int_{\hat{h}}^{1}(-2 \pi \log \hat{\rho}) \hat{h} \cos \theta \hat{\rho} \mathrm{d} \rho\right] \\
& =\frac{\pi q}{p} \cos \theta\left(\frac{\hat{h}^{3}}{4}-\frac{\hat{h}}{4}\right) .
\end{aligned}
$$

For $\tilde{B}_{2}$ we note that

$$
\begin{aligned}
& \tilde{B}_{2}=\frac{q}{2 p} \int_{0}^{1} \int_{0}^{2 \pi} \log \frac{1}{\sqrt{\hat{h}^{2}+\hat{\rho}^{2}-2 \hat{h} \hat{\rho} \cos (\theta-\omega)}} \hat{\rho} \cos \omega \hat{\rho} \mathrm{d} \omega \mathrm{d} \hat{\rho} \\
& =\frac{q}{2 p} \int_{0}^{1} \int_{0}^{2 \pi} \log \frac{1}{\sqrt{\hat{h}^{2}+\hat{\rho}^{2}-2 \hat{h} \hat{\rho} \cos (\theta-\omega)}} \hat{\rho} \cos (\theta-\omega) \cos \theta \hat{\rho} \mathrm{d} \omega \mathrm{d} \hat{\rho} \\
& +\frac{q}{2 p} \int_{0}^{1} \int_{0}^{2 \pi} \log \frac{1}{\sqrt{\hat{h}^{2}+\hat{\rho}^{2}-2 \hat{h} \hat{\rho} \cos (\theta-\omega)}} \hat{\rho} \sin (\theta-\omega) \sin \theta \hat{\rho} \mathrm{d} \omega \mathrm{d} \hat{\rho} \\
& =\frac{q}{2 p} \int_{0}^{1} \int_{0}^{2 \pi} \log \frac{1}{\sqrt{\hat{h}^{2}+\hat{\rho}^{2}-2 \hat{h} \hat{\rho} \cos (\theta-\omega)}} \hat{\rho} \cos (\theta-\omega) \cos \theta \hat{\rho} \mathrm{d} \omega \mathrm{d} \hat{\rho} .
\end{aligned}
$$

To reach the last line, we have deduced from the absence of $\sin n \omega$ terms in 3.6 that

$$
\frac{q}{2 p} \int_{0}^{1} \int_{0}^{2 \pi} \log \frac{1}{\sqrt{\hat{h}^{2}+\hat{\rho}^{2}-2 \hat{h} \hat{\rho} \cos (\theta-\omega)}} \hat{\rho} \sin (\theta-\omega) \sin \theta \hat{\rho} \mathrm{d} \omega \mathrm{d} \hat{\rho}=0 .
$$

Another consequence of 3.6 is

$$
\begin{aligned}
& \int_{0}^{2 \pi} \log \frac{1}{\sqrt{\hat{h}^{2}+\hat{\rho}^{2}-2 \hat{h} \hat{\rho} \cos (\theta-\omega)}} \cos (\theta-\omega) \mathrm{d} \omega \\
& \quad=\int_{0}^{2 \pi} \log \frac{1}{\sqrt{1+\beta^{2}-2 \beta \cos \omega}} \cos \omega \mathrm{d} \omega \\
& \quad=\int_{0}^{2 \pi} \beta \cos ^{2} \omega \mathrm{d} \omega=\pi \beta \quad \text { where } \beta= \begin{cases}\hat{\rho} / \hat{h} & \text { if } \hat{\rho}<\hat{h}, \\
\hat{h} / \hat{\rho} & \text { if } \hat{\rho} \geqslant \hat{h} .\end{cases}
\end{aligned}
$$

Therefore

$$
\tilde{B}_{2}=\frac{\pi q}{2 p}\left[\int_{0}^{\hat{h}} \frac{\hat{\rho}}{\hat{h}} \hat{\rho}^{2} \cos \theta \mathrm{d} \hat{\rho}+\int_{\hat{h}}^{1} \frac{\hat{h}}{\hat{\rho}} \hat{\rho}^{2} \cos \theta \mathrm{d} \hat{\rho}\right]=\frac{\pi q}{2 p} \cos \theta\left(\frac{\hat{h}}{2}-\frac{\hat{h}^{3}}{4}\right)
$$

Following (3.9) and 3.10) we deduce

$$
\tilde{B}=\frac{\pi q}{8 p} \hat{h}^{3} \cos \theta+O\left(\frac{q^{2}}{p^{2}}\right)
$$


By (3.5), 3.8, and (3.11) we find

$$
I_{I I}=q^{2}\left(\frac{1}{4}-\frac{\hat{h}^{2}}{4}\right)+\frac{q^{3}}{16 p} \hat{h}^{3} \cos \theta+O\left(\frac{q^{4}}{p^{2}}\right) .
$$

The estimation of $I_{I I}$ is straightforward. Because $G_{3}(\cdot, \cdot, s, t)$ is in $C^{2, \alpha}\left(\mathbb{R}_{+}^{2}\right)$ and $D_{2} G_{3}(1,0,1,0)=0$ according to Lemma 2.2(iii),

$$
\begin{aligned}
I_{I I}= & q^{2} \int_{0}^{1} \int_{0}^{2 \pi} G_{3}\left(1+\frac{q}{p} \hat{h} \cos \theta, \frac{q}{p} \hat{h} \sin \theta, 1+\frac{q}{p} \hat{\rho} \cos \omega, \frac{q}{p} \hat{\rho} \sin \omega\right) \hat{\rho} \mathrm{d} \omega \mathrm{d} \hat{\rho} \\
= & q^{2} \int_{0}^{1} \int_{0}^{2 \pi}\left[G_{3}(1,0,1,0)+D_{1} G_{3}(1,0,1,0) \frac{q}{p} \hat{h} \cos \theta+D_{2} G_{3}(1,0,1,0) \frac{q}{p} \hat{h} \sin \theta\right. \\
& \left.+D_{3} G_{3}(1,0,1,0) \frac{q}{p} \hat{\rho} \cos \omega+D_{4} G_{3}(1,0,1,0) \frac{q}{p} \hat{\rho} \sin \omega+O\left(\frac{q^{2}}{p^{2}}\right)\right] \hat{\rho} \mathrm{d} \omega \mathrm{d} \hat{\rho} \\
= & q^{2} \int_{0}^{1} \int_{0}^{2 \pi}\left[G_{3}(1,0,1,0)+D_{1} G_{3}(1,0,1,0) \frac{q}{p} \hat{h} \cos \theta\right] \hat{\rho} \mathrm{d} \omega \mathrm{d} \hat{\rho}+O\left(\frac{q^{4}}{p^{2}}\right) \\
= & \pi q^{2} G_{3}(1,0,1,0)+\frac{\pi q^{3}}{p} D_{1} G_{3}(1,0,1,0) \hat{h} \cos \theta+O\left(\frac{q^{4}}{p^{2}}\right) .
\end{aligned}
$$

We now combine (3.4), 3.12, and (3.13) to conclude that

$$
\begin{aligned}
\mathcal{N}\left(E_{0}\right)(p+h \cos \theta, h \sin \theta)= & \frac{q^{2}}{2} \log \frac{p}{q}+q^{2}\left[\frac{1}{4}-\frac{\hat{h}^{2}}{4}+\pi G_{3}(1,0,1,0)\right] \\
& -\frac{q^{3}}{4 p}\left(\log \frac{p}{q}\right) \hat{h} \cos \theta+\frac{q^{3}}{p}\left[\frac{\hat{h}^{3}}{16}+\pi D_{1} G_{3}(1,0,1,0) \hat{h}\right] \cos \theta \\
& +O\left(\frac{q^{4}}{p^{2}} \log \frac{p}{q}\right) .
\end{aligned}
$$

Since

and

$$
G_{3}(p, 0, p, 0)=G_{3}(1,0,1,0)-\frac{1}{2 \pi} \log \frac{1}{p}
$$

$$
p D_{1} G_{3}(p, 0, p, 0)=D_{1} G_{3}(1,0,1,0)+\frac{1}{4 \pi} \log \frac{1}{p}
$$

by (3.3), the lemma follows from (3.14).

We insert $E_{0}$ into the left side of the equation $\mathcal{H}(\partial E)+\gamma \mathcal{N}(E)=\lambda$ and see how much it deviates from a constant.

LEMMA 3.2 On $\partial E_{0}$,

$$
\begin{aligned}
\left(\mathcal{H}\left(\partial E_{0}\right)\right. & \left.+\gamma \mathcal{N}\left(E_{0}\right)\right)(p+q \cos \theta, q \sin \theta) \\
= & \frac{1}{2 q}+\frac{\cos \theta}{2 p}+O\left(\frac{q}{p^{2}}\right)+\gamma\left\{\frac{q^{2}}{2} \log \frac{1}{q}+\pi q^{2} G_{3}(p, 0, p, 0)\right. \\
& \left.-\frac{q^{3}}{4 p}\left(\log \frac{1}{q}\right) \cos \theta+\frac{q^{3}}{p}\left[\pi p D_{1} G_{3}(p, 0, p, 0)+\frac{1}{16}\right] \cos \theta+O\left(\frac{q^{4}}{p^{2}} \log \frac{p}{q}\right)\right\} .
\end{aligned}
$$


Proof. The mean curvature, as the average of the principal curvatures, of the torus $\partial E_{0}$ at $(r, z)=$ $(p+q \cos \theta, q \sin \theta)$ is

$$
\mathcal{H}\left(\partial E_{0}\right)(p+q \cos \theta, q \sin \theta)=\frac{1}{2}\left(\frac{1}{q}+\frac{\cos \theta}{p+q \cos \theta}\right)=\frac{1}{2 q}+\frac{\cos \theta}{2 p}+O\left(\frac{q}{p^{2}}\right) .
$$

Lemma 3.2 follows from 3.15 and Lemma 3.1 .

We proceed to estimate $\mathcal{J}\left(E_{0}\right)$.

LEMMA 3.3

$$
\mathcal{J}\left(E_{0}\right)=2 \pi^{2} p q+\frac{\gamma}{2}\left(\pi^{2} p q^{4} \log \frac{1}{q}+\frac{\pi^{2} p q^{4}}{4}+2 \pi^{3} p q^{4} G_{1}(p, 0, p, 0)\right)+O\left(\frac{\gamma q^{4}}{p^{2}} \log \frac{p}{q}\right) .
$$

Proof. The perimeter of $E_{0}$ is

$$
\mathcal{P}\left(E_{0}\right)=\sum_{j=1}^{2} 4 \pi^{2} p q
$$

which is the area of $\partial E_{0}$.

The second part of $\mathcal{J}\left(E_{0}\right)$ is $\gamma / 2$ times

$$
\begin{array}{rl}
2 \pi \int_{E_{0}} \mathcal{N}\left(E_{0}\right)(r, z) r & \mathrm{~d} r \mathrm{~d} z \\
& =2 \pi \int_{0}^{q} \int_{0}^{2 \pi} \mathcal{N}\left(E_{0}\right)(p+h \cos \theta, h \sin \theta)(p+h \cos \theta) h \mathrm{~d} \theta \mathrm{d} h .
\end{array}
$$

By Lemma 3.1.

$$
\begin{aligned}
\mathcal{N}\left(E_{0}\right)(p+h \cos \theta, h \sin \theta)=\frac{q^{2}}{2} \log \frac{1}{q}+q^{2}\left[\pi G_{3}(p, 0, p, 0)+\frac{1}{4}-\frac{h^{2}}{4 q^{2}}\right] \\
-\frac{q^{3}}{4 p}\left(\log \frac{1}{q}\right) \frac{h}{q} \cos \theta+\frac{q^{3}}{p}\left[\pi p D_{1} G_{3}(p, 0, p, 0) \frac{h}{q}+\frac{h^{3}}{16 q^{3}}\right] \cos \theta+O\left(\frac{q^{4}}{p^{2}} \log \frac{p}{q}\right) .
\end{aligned}
$$

Therefore 3.17) becomes

$2 \pi \int_{E_{0}} \mathcal{N}\left(E_{0}\right)(r, z) r \mathrm{~d} r \mathrm{~d} z$

$$
\begin{aligned}
= & 2 \pi \int_{0}^{q} \int_{0}^{2 \pi}\left[\frac{q^{2}}{2} \log \frac{1}{q}+q^{2}\left(\pi G_{3}(p, 0, p, 0)+\frac{1}{4}-\frac{h^{2}}{4 q^{2}}\right)\right](p+h \cos \theta) h \mathrm{~d} \theta \mathrm{d} h \\
& +2 \pi \int_{0}^{q} \int_{0}^{2 \pi}\left[-\frac{q^{3}}{4 p}\left(\log \frac{1}{q}\right) \frac{h}{q} \cos \theta\right. \\
& \left.+\frac{q^{3}}{p}\left(\pi p D_{1} G_{3}(p, 0, p, 0) \frac{h}{q}+\frac{h^{3}}{16 q^{3}}\right) \cos \theta\right](p+h \cos \theta) h \mathrm{~d} \theta \mathrm{d} h \\
& +O\left(\frac{q^{4}}{p^{2}} \log \frac{p}{q}\right)
\end{aligned}
$$




$$
\begin{aligned}
& =\pi^{2} p q^{4} \log \frac{1}{q}+\frac{\pi^{2} p q^{4}}{4}+2 \pi^{3} p q^{4} G_{3}(p, 0, p, 0)+O\left(\frac{q^{6}}{p} \log \frac{p}{q}\right)+O\left(\frac{q^{4}}{p^{2}} \log \frac{p}{q}\right) \\
& =\pi^{2} p q^{4} \log \frac{1}{q}+\frac{\pi^{2} p q^{4}}{4}+2 \pi^{3} p q^{4} G_{3}(p, 0, p, 0)+O\left(\frac{q^{4}}{p^{2}} \log \frac{p}{q}\right) .
\end{aligned}
$$

The lemma follows from (3.16) and (3.18), since $G_{3}(p, 0, p, 0)=G_{1}(p, 0, p, 0)$ by Lemma 2.1.

At this point we can guess what the sizes of $p$ and $q$ should be for $E_{0}$ to be a good approximate solution. We want the right side of the estimate in Lemma 3.2 to be as close to a constant as possible. Therefore the $\cos \theta$ dependent terms should cancel out. Note that these terms are

$$
\frac{\cos \theta}{2 p}+\gamma\left\{-\frac{q^{3}}{4 p}\left(\log \frac{1}{q}\right) \cos \theta+\frac{q^{3}}{p}\left[\pi p D_{1} G_{3}(p, 0, p, 0)+\frac{1}{16}\right] \cos \theta\right\} .
$$

As we know $p D_{1} G_{3}(p, 0, p, 0)=D_{1} G_{3}(1,0,1,0)+\frac{1}{4 \pi} \log \frac{1}{p}$ by the proof of Lemma 3.1 the above is

$$
\frac{\cos \theta}{2 p}+\gamma\left\{-\frac{q^{3}}{4 p}\left(\log \frac{p}{q}\right) \cos \theta+\frac{q^{3}}{p}\left[\pi D_{1} G_{3}(1,0,1,0)+\frac{1}{16}\right] \cos \theta\right\} .
$$

The leading order of this quantity is

$$
\frac{\cos \theta}{2 p}-\frac{\gamma q^{3}}{4 p}\left(\log \frac{p}{q}\right) \cos \theta
$$

So to be a good approximate solution, $E_{0}$ should satisfy

$$
\frac{1}{2 p}-\frac{\gamma q^{3}}{4 p} \log \frac{p}{q} \approx 0
$$

By 3.1, 3.19] becomes

$$
\gamma \approx \frac{2}{3 q^{3} \log \frac{1}{q}}
$$

Comparing this with the definition (1.5) of the function $f$, we find that for $E_{0}$ to be a good approximate solution we must have

$$
q \approx f(\gamma) .
$$

Motivated by 3.20 from now on we require that the cross section radius $q$ of our approximate solution satisfy

$$
q \in[f(\gamma) / 2,2 f(\gamma)]
$$

Consequently, $p=\frac{1}{2 \pi^{2} q^{2}}$ satisfies

$$
p \in\left[\frac{1}{8 \pi^{2} f^{2}(\gamma)}, \frac{2}{\pi^{2} f^{2}(\gamma)}\right] .
$$

Note that

$$
q \rightarrow 0 \quad \text { and } \quad p \rightarrow \infty \quad \text { as } \gamma \rightarrow \infty
$$




\section{Perturbation to $E_{0}$}

The exact solution will be found near an approximate solution $E_{0}$. For this reason we need a way to perturb $E_{0}$. Let $u=u(\theta)$ be a $2 \pi$-periodic positive function and define a set

$$
E=\bigcup_{\theta \in[0,2 \pi]}\{(p+h \cos \theta, h \sin \theta): h \in[0, u(\theta)]\}
$$

in $\mathbb{R}_{+}^{2}$. Corresponding to $E$ there is an axisymmetric set in $\mathbb{R}^{3}$, which we again denote by $E$. If $u(\theta)=q$ for all $\theta \in[0,2 \pi], E$ becomes our torus $E_{0}$ considered before. When $u(\theta)$ is close to $q$ for all $\theta \in[0,2 \pi], E$ is a perturbation of $E_{0}$.

Once the set $E$ is described by $u, \mathcal{J}(E)$ can be expressed in terms of $u$ :

$$
\mathcal{J}(E)=\pi \int_{0}^{2 \pi}(p+u(\theta) \cos \theta) \sqrt{\left(u^{\prime}(\theta)\right)^{2}+(u(\theta))^{2}} \mathrm{~d} \theta+\gamma \pi \int_{E} \mathcal{N}(E)(r, z) r \mathrm{~d} r \mathrm{~d} z
$$

where the first term gives half the area of $\partial E$ and the second term is the nonlocal part of $\mathcal{J}(E)$.

It is often more convenient to introduce another variable $v$ in place of $u$. The constraint (1.4) requires

$$
\begin{aligned}
1 & =2 \pi \int_{E} r \mathrm{~d} r \mathrm{~d} z=2 \pi \int_{0}^{2 \pi} \int_{0}^{u(\theta)}(p+\rho \cos \theta) \rho \mathrm{d} \rho \mathrm{d} \theta \\
& =2 \pi \int_{0}^{2 \pi}\left(\frac{p u^{2}(\theta)}{2}+\frac{u^{3}(\theta) \cos \theta}{3}\right) \mathrm{d} \theta .
\end{aligned}
$$

We set

$$
v(\theta)=\frac{p u^{2}(\theta)}{2}+\frac{u^{3}(\theta) \cos \theta}{3} .
$$

The set $E$ is now specified by the function $v$. With respect to $v$ by (4.1) the constraint (1.4) takes the form

$$
\int_{0}^{2 \pi} v(\theta) \mathrm{d} \theta=\frac{1}{2 \pi}
$$

which is simpler than 4.1 . Consequently, $\mathcal{J}$ becomes a functional of $v: \mathcal{J}=\mathcal{J}(v)$. The variation of $\mathcal{J}$ is written as

$$
\mathcal{H}(v)+\gamma \mathcal{N}(v)
$$

where $\mathcal{H}(v)$ is the variation, with respect to $v$, of the first term of $\mathcal{J}$ and $\gamma \mathcal{N}(v)$ is the variation of the second term of $\mathcal{J}$. More precisely let $\phi=\phi(\theta)$ be a $2 \pi$-periodic function satisfying $\int_{0}^{2 \pi} \phi(\theta) \mathrm{d} \theta=0$. Then

$$
\mathcal{J}^{\prime}(v)(\phi)=\left.\frac{\mathrm{d} \mathcal{J}(v+\epsilon \phi)}{\mathrm{d} \epsilon}\right|_{\epsilon=0}=2 \pi \int_{0}^{2 \pi}(\mathcal{H}(v)+\gamma \mathcal{N}(v)) \phi \mathrm{d} \theta .
$$

Both $\mathcal{H}(v)$ and $\mathcal{N}(v)$ are functions of $\theta$.

The reader may have noticed that we used the same $\mathcal{H}$ to denote the mean curvature operator of the surface $\partial E$ earlier, and an operator on a $2 \pi$-periodic function $v$ now. This abuse of notation is deliberate. $\mathcal{H}(\partial E)$ and $\mathcal{H}(v)$ stand for the same mean curvature of the boundary of the set $E$ 
described by $v$. Similarly $\mathcal{N}(E)$ and $\mathcal{N}(v)$ are the same Newtonian potential of the set $E$ described by $v$. The equation (1.1) now becomes

$$
\mathcal{H}(v)+\gamma \mathcal{N}(v)=\lambda .
$$

Since the same set $E$ can be described by either the variable $u$ or the variable $v$, to avoid confusion we will call $u$ the radius variable of the set $E$ and $v$ the volume variable of the set $E$. The approximate solution $E_{0}$ described by the radius variable is

$$
u(\theta)=q .
$$

We denote the corresponding volume variable for $E_{0}$ by $\psi$, given by

$$
\psi(\theta)=\frac{p q^{2}}{2}+\frac{q^{3} \cos \theta}{3} .
$$

Note that unlike the radius variable $u(\theta)=q$, the volume variable $\psi$ is not a constant function.

Note that if we integrate (4.2) with respect to $\theta$, then

$$
\lambda=\frac{1}{2 \pi} \int_{0}^{2 \pi}(\mathcal{H}(v)+\gamma \mathcal{N}(v)) \mathrm{d} \theta .
$$

Introduce the notion of average

$$
\overline{\mathcal{H}(v)}=\frac{1}{2 \pi} \int_{0}^{2 \pi} \mathcal{H}(v) \mathrm{d} \theta, \quad \overline{\mathcal{N}(v)}=\frac{1}{2 \pi} \int_{0}^{2 \pi} \mathcal{N}(v) \mathrm{d} \theta .
$$

Also introduce the operator $\mathcal{S}$ by

$$
\mathcal{S}(v)=\mathcal{H}(v)-\overline{\mathcal{H}(v)}+\gamma \mathcal{N}(v)-\gamma \overline{\mathcal{N}(v)} .
$$

Then (4.2) becomes

$$
\mathcal{S}(v)=0 .
$$

For $\mathcal{S}$ to be meaningful, we must specify its domain. Recall $H^{2}\left(S^{1}\right)$, the $W^{2,2}$ Sobolev space of $2 \pi$-periodic functions. Let

$$
\mathcal{X}=\left\{v \in H^{2}\left(S^{1}\right): \int_{0}^{2 \pi} v(\theta) \mathrm{d} \theta=\frac{1}{2 \pi}\right\}
$$

be the metric space equipped with the $H^{2}\left(S^{1}\right)$ norm. Note that $\psi \in \mathcal{X}$. Our $\mathcal{S}$ is defined on the $v$ 's which are in a neighborhood of $\psi \in \mathcal{X}$. We often write $v=\psi+\phi$. Let

$$
\mathcal{X}^{\prime}=\left\{\phi \in H^{2}\left(S^{1}\right): \int_{0}^{2 \pi} \phi(\theta) \mathrm{d} \theta=0\right\} .
$$

Then $\phi$ is in a neighborhood of $0 \in \mathcal{X}^{\prime}$. The target space of the operator $\mathcal{S}$ is

$$
\mathcal{Y}=\left\{g \in L^{2}\left(S^{1}\right): \int_{0}^{2 \pi} g(\theta) \mathrm{d} \theta=0\right\} .
$$


Next we analyze $\mathcal{S}^{\prime}(\psi)$ where $\mathcal{S}^{\prime}$ stands for the Fréchet derivative of $\mathcal{S}$. At $\psi, \mathcal{S}^{\prime}(\psi)$ is a linear operator from $\mathcal{X}^{\prime}$ to $\mathcal{Y}$. This operator will be written as the sum of a major part and a minor part denoted by $\mathcal{M}$ and $\widetilde{\mathcal{M}}$ respectively.

To identify $\mathcal{M}$ we treat the two parts of $\mathcal{S}, \mathcal{H}(v)-\overline{\mathcal{H}(v)}$ and $\mathcal{N}(v)-\overline{\mathcal{N}(v)}$, separately. The first part of $\mathcal{S}$ comes from the perimeter part of $\mathcal{J}$ which is

$$
\pi \int_{0}^{2 \pi}(p+u \cos \theta) \sqrt{\left(u^{\prime}\right)^{2}+u^{2}} \mathrm{~d} \theta
$$

when the radius variable $u$ is used. This is to be converted to an integral with respect to the volume variable $v$. Let

$$
A(u, \theta)=\frac{p u^{2}}{2}+\frac{u^{3} \cos \theta}{3} .
$$

Then

$$
v(\theta)=A(u(\theta), \theta) .
$$

Denote the inverse of $A$, as a function of $u$ with $\theta$ held fixed, by $B=B(v, \theta)$ such that

$$
u(\theta)=B(v(\theta), \theta) .
$$

Define the Lagrangian $L$ from $\mathbb{R} \times \mathbb{R}_{+} \times S^{1}$ to $\mathbb{R}$, with respect to the volume variable, by

$$
L(\dot{v}, v, \theta)=(p+u \cos \theta) \sqrt{\dot{u}^{2}+u^{2}},
$$

where $(\dot{u}, u, \theta)$ and $(\dot{v}, v, \theta)$ transform to each other according the rules

$$
\left(\begin{array}{l}
\dot{u} \\
u \\
\theta
\end{array}\right) \mapsto\left(\begin{array}{l}
\dot{v} \\
v \\
\theta
\end{array}\right)=\left(\begin{array}{l}
\dot{u} A_{u}(u, \theta)+A_{\theta}(u, \theta) \\
A(u, \theta) \\
\theta
\end{array}\right)
$$

and

$$
\left(\begin{array}{l}
\dot{v} \\
v \\
\theta
\end{array}\right) \mapsto\left(\begin{array}{l}
\dot{u} \\
u \\
\theta
\end{array}\right)=\left(\begin{array}{l}
\dot{v} B_{v}(v, \theta)+B_{\theta}(v, \theta) \\
B(v, \theta) \\
\theta
\end{array}\right) .
$$

Following (4.3) and (4.4) we find that as $p \rightarrow \infty$,

$$
p^{-1 / 2} L(\dot{v}, v, \theta) \rightarrow \sqrt{\frac{\dot{v}^{2}}{2 v}+2 v}
$$

in $C_{\mathrm{loc}}^{\infty}\left(\mathbb{R} \times \mathbb{R}_{+} \times S^{1}\right)$, and

$$
\psi(\theta) \rightarrow \frac{1}{4 \pi^{2}}
$$

in $C^{\infty}\left(S^{1}\right)$. The second derivative of the functional

$$
v \mapsto \frac{1}{2} \int_{0}^{2 \pi} \sqrt{\frac{\left(v^{\prime}(\theta)\right)^{2}}{2 v(\theta)}+2 v(\theta)} \mathrm{d} \theta
$$


at $v=\frac{1}{4 \pi^{2}}$ is the linear operator

$$
\phi \in \mathcal{X}^{\prime} \mapsto-\sqrt{2} \pi^{3}\left(\phi^{\prime \prime}+\phi\right) \in \mathcal{Y} .
$$

This gives us the first part of $\mathcal{M}$ :

$$
\phi \in \mathcal{X}^{\prime} \mapsto-\sqrt{2} \pi^{3} p^{1 / 2}\left(\phi^{\prime \prime}+\phi\right) \in \mathcal{Y} .
$$

The second part of $\mathcal{M}$ comes from the Fréchet derivative of $\mathcal{N}$. Let $u=B(\psi+\epsilon \phi)$ and compute

$$
\begin{aligned}
& \left.\frac{\partial \mathcal{N}(B(\psi+\epsilon \phi))}{\partial \epsilon}\right|_{\epsilon=0} \\
& =\int_{0}^{2 \pi} G(p+q \cos \theta, q \sin \theta, p+q \cos \omega, p+q \sin \omega) q D_{1} B(\psi(\omega), \omega) \phi(\omega) \mathrm{d} \omega \\
& +\phi(\theta) D_{1} B(\psi(\theta), \theta) \int_{E_{0}}\left(D_{1} G(p+q \cos \theta, q \sin \theta, s, t), D_{2} G(p+q \cos \theta, q \sin \theta, s, t)\right) \cdot e^{i \theta} \mathrm{d} s \mathrm{~d} t
\end{aligned}
$$

Some smaller terms are ignored at this point. First note that

$$
D_{1} B(\psi(\theta), \theta)=\frac{1}{p q+q^{2} \cos \theta} .
$$

We will replace $D_{1} B(\psi)$ by $\frac{1}{p q}$. Second we replace $G(r, z, s, t)$ by $\frac{1}{2 \pi} \log \frac{1}{|(r, z)-(s, t)|}$ because of Lemma 2.1. Then 4.6 is replaced by the outcome of the function

$$
\phi \mapsto \frac{1}{p} \int_{0}^{2 \pi} \frac{1}{2 \pi} \log \frac{1}{q\left|e^{i \theta}-e^{i \omega}\right|} \phi(\omega) \mathrm{d} \omega-\frac{\phi(\theta)}{2 \pi p q} \int_{0}^{2 \pi} \int_{0}^{q} \frac{q e^{i \theta}-\rho e^{i \omega}}{\left|q e^{i \theta}-\rho e^{i \omega}\right|^{2}} \cdot e^{i \theta} \rho \mathrm{d} \rho \mathrm{d} \omega .
$$

The double integral in 4.8 may be evaluated:

$$
\int_{0}^{2 \pi} \int_{0}^{q} \frac{q e^{i \theta}-\rho e^{i \omega}}{\left|q e^{i \theta}-\rho e^{i \omega}\right|^{2}} \cdot e^{i \theta} \rho \mathrm{d} \rho \mathrm{d} \omega=q \pi .
$$

Then 4.8 becomes

$$
\phi \mapsto \frac{1}{2 \pi p} \log \frac{1}{q} \int_{0}^{2 \pi} \phi(\omega) \mathrm{d} \omega+\frac{1}{p} \int_{0}^{2 \pi} \frac{1}{2 \pi} \log \frac{1}{\mid e^{i \theta}-e^{i \omega \mid}} \phi(\omega) \mathrm{d} \omega-\frac{\phi(\theta)}{2 p} .
$$

Since our $\phi$ is in $\mathcal{X}^{\prime}$ where $\int_{0}^{2 \pi} \phi(\omega) \mathrm{d} \omega=0$, the first term in 4.10 vanishes and 4.10 becomes

$$
\phi \in \mathcal{X}^{\prime} \mapsto \frac{1}{p} \int_{0}^{2 \pi} \frac{1}{2 \pi} \log \frac{1}{\mid e^{i \theta}-e^{i \omega \mid}} \phi(\omega) \mathrm{d} \omega-\frac{\phi(\theta)}{2 p} \in \mathcal{Y} .
$$

Combining 4.5 and 4.11) we find that $\mathcal{H}^{\prime}(\psi)-\overline{\mathcal{H}^{\prime}(\psi)}$ is dominated by

$$
\phi \in \mathcal{X}^{\prime} \mapsto-\sqrt{2} \pi^{3} p^{1 / 2}\left(\phi^{\prime \prime}+\phi\right) \in \mathcal{Y},
$$


and $\gamma \mathcal{N}^{\prime}(\psi)-\gamma \overline{\mathcal{N}^{\prime}(\psi)}$ is dominated by

$$
\phi \in \mathcal{X}^{\prime} \mapsto \frac{\gamma}{p} \int_{0}^{2 \pi} \frac{1}{2 \pi} \log \frac{1}{\left|e^{i \theta}-e^{i \omega}\right|} \phi(\omega) \mathrm{d} \omega-\frac{\gamma \phi(\theta)}{2 p} \in \mathcal{Y} .
$$

However $p^{1 / 2} \gg \gamma / p$ by 1.5 and 3.22 . The operator 4.13 is negligible compared to 4.12 . We have identified $\mathcal{M}$ :

$$
\phi \in \mathcal{X}^{\prime} \mapsto \mathcal{M}(\phi)=-\sqrt{2} \pi^{3} p^{1 / 2}\left(\phi^{\prime \prime}+\phi\right) \in \mathcal{Y} .
$$

The rest of $\mathcal{S}^{\prime}(\psi)$ is denoted by $\widetilde{\mathcal{M}}$ so that

$$
\mathcal{S}^{\prime}(\psi)=\mathcal{M}+\widetilde{\mathcal{M}}
$$

The eigenpairs of $\mathcal{M}$ are

$$
2^{5 / 2} \pi^{3} p^{1 / 2}\left(n^{2}-1\right) ; \quad \cos n \theta, \sin n \theta ; \quad n=1,2,3, \ldots
$$

Note that the functions in $\mathcal{X}^{\prime}$ have zero average, so the $n=0$ mode is not included in 4.16. All eigenvalues in (4.16) are positive except for the mode $n=1$ where 0 is an eigenvalue and $\cos \theta$ and $\sin \theta$ are the corresponding eigenfunctions. To proceed we remove $\cos \theta$ and $\sin \theta$ from $\mathcal{X}^{\prime}$ and set

$$
\mathcal{X}_{*}^{\prime}=\left\{\phi \in \mathcal{X}^{\prime}: \phi \perp \cos \theta, \phi \perp \sin \theta\right\}, \quad \mathcal{Y}_{*}=\{\phi \in \mathcal{Y}: \phi \perp \cos \theta, \phi \perp \sin \theta\} .
$$

Let $\Pi$ be the orthogonal projection operator

$$
\Pi: \mathcal{Y} \rightarrow \mathcal{Y}_{*}
$$

and consider the operator

$$
\Pi \mathcal{S}^{\prime}(\psi): \mathcal{X}_{*}^{\prime} \rightarrow \mathcal{Y}_{*}
$$

The following lemma summarizes the properties of $\Pi \mathcal{S}^{\prime}(\psi): \mathcal{X}_{*}^{\prime} \rightarrow \mathcal{Y}_{*}$, whose proof which we omit is similar to that of Lemma 3.1 of [33].

LEMmA 4.1 (i) There exists $C>0$ independent of $p, q$ and $\gamma$ such that

$$
\|\phi\|_{H^{2}} \leqslant C p^{-1 / 2}\left\|\Pi \mathcal{S}^{\prime}(\psi) \phi\right\|_{L^{2}}
$$

for all $\phi \in \mathcal{X}_{*}^{\prime}$. Moreover the operator $\Pi \mathcal{S}^{\prime}(\psi)$ is one-to-one and onto from $\mathcal{X}_{*}^{\prime}$ to $\mathcal{Y}_{*}$.

(ii) There exists $C>0$ independent of $p, q$ and $\gamma$ such that for all $\phi \in \mathcal{X}_{*}^{\prime}$,

$$
\|\phi\|_{H^{1}}^{2} \leqslant C p^{-1 / 2}\left\langle\Pi \mathcal{S}^{\prime}(\psi) \phi, \phi\right\rangle .
$$

The second Fréchet derivative of $\mathcal{S}$ is estimated in the next lemma. The proof is similar to that of Lemma 3.2 of [33] which we again omit.

LEMMA 4.2 There exists $C>0$ independent of $p, q$ and $\gamma$ such that

$$
\left\|\mathcal{S}^{\prime \prime}(v)\left(\phi_{1}, \phi_{2}\right)\right\|_{L^{2}} \leqslant C p^{1 / 2}\|u\|_{H^{2}}\|v\|_{H^{2}}
$$

for all $v$ near $\psi$, in the sense $\|v-\psi\|_{H^{2}}<\frac{1}{2}\|\psi\|_{H^{2}}$, and all $\phi_{1}, \phi_{2} \in \mathcal{X}^{\prime}$. 


\section{Reduction}

The nonlinear operator $\mathcal{S}$ maps a neighborhood of $\psi$ in $\mathcal{X}$ to $\mathcal{Y}$. In this section it will be proved that, for each $p \in\left[\frac{1}{8 \pi^{2} f^{2}(\gamma)}, \frac{2}{\pi^{2} f^{2}(\gamma)}\right]$, there exists a function $\varphi \in \mathcal{X}_{*}^{\prime}$ such that

$$
\mathcal{S}(\psi+\varphi)(\theta)=A_{1} \cos \theta+A_{2} \sin \theta
$$

for some $A_{1}, A_{2} \in \mathbb{R}$. The equation (5.1) may be written as

$$
\Pi \mathcal{S}(\psi+\varphi)=0
$$

where $\Pi$ is given in 4.17). In the next section we will find a particular $p$, say $p_{\gamma}$, such that at $p=p_{\gamma}, A_{1}=A_{2}=0$. This means that by finding $\varphi$ one reduces the original infinite-dimensional problem 1.1 to a one-dimensional problem of finding $p_{\gamma}$ in the interval $\left[\frac{1}{8 \pi^{2} f^{2}(\gamma)}, \frac{2}{\pi^{2} f^{2}(\gamma)}\right]$.

LEMMA 5.1 For every $p \in\left[\frac{1}{8 \pi^{2} f^{2}(\gamma)}, \frac{2}{\pi^{2} f^{2}(\gamma)}\right]$, there exists $\varphi \in \mathcal{X}_{*}^{\prime}$ such that $\varphi$ solves 5.2 and $\|\varphi\|_{H^{2}} \leqslant M f^{6}(\gamma)$ where $M$ is a sufficiently large constant independent of $\gamma$ and $p$.

Proof. Expand $\mathcal{S}(\psi+\phi)$ as

$$
\mathcal{S}(\psi+\phi)=\mathcal{S}(\psi)+\mathcal{S}^{\prime}(\psi)(\phi)+\mathcal{R}(\phi)
$$

where $\mathcal{R}$ is a higher order term defined by (5.3). Rewrite 5.2 in a fixed point form:

$$
\phi=-\left(\Pi \mathcal{S}^{\prime}(\psi)\right)^{-1}(\Pi \mathcal{S}(\psi)+\Pi \mathcal{R}(\phi)) \text {. }
$$

To use the contraction mapping principle in the fixed point setting (5.4), let

$$
\mathcal{T}(\phi)=-\left(\Pi \mathcal{S}^{\prime}(\psi)\right)^{-1}(\Pi \mathcal{S}(\psi)+\Pi \mathcal{R}(\phi))
$$

be an operator defined on

$$
D(\mathcal{T})=\left\{\phi \in \mathcal{X}_{*}^{\prime}:\|\phi\|_{H^{2}} \leqslant M f^{6}(\gamma)\right\}
$$

where the constant $M$ is sufficiently large and will be made more precise later. Note that when $\gamma$ is sufficiently large, every $\phi \in D(\mathcal{T})$ is very small compared with $\psi$, and hence $\mathcal{T}$ is well-defined.

Lemma 3.2 (3.21), and (3.22) assert that

$$
\begin{aligned}
\mathcal{H}(\psi)+\gamma \mathcal{N}(\psi)= & \frac{1}{2 q}+\frac{\cos \theta}{2 p}+\gamma\left[\frac{q^{2}}{2} \log \frac{1}{q}+\pi q^{2} G_{3}(p, 0, p, 0)-\frac{q^{3}}{4 p}\left(\log \frac{1}{q}\right) \cos \theta\right. \\
& \left.+\frac{q^{3}}{p}\left(\pi p D_{1} G_{3}(p, 0, p, 0)+\frac{1}{16}\right) \cos \theta\right]+O\left(f^{5}(\gamma)\right) .
\end{aligned}
$$

Taking the average of 5.7 we find

$$
\overline{\mathcal{H}(\psi)}+\gamma \overline{\mathcal{N}(\psi)}=\frac{1}{2 q}+\gamma\left[\frac{q^{2}}{2} \log \frac{1}{q}+\pi q^{2} G_{3}(p, 0, p, 0)\right]+O\left(f^{5}(\gamma)\right)
$$


Subtract (5.8) from 5.7) to obtain

$$
\mathcal{S}(\psi)=\frac{\cos \theta}{2 p}+\gamma\left[-\frac{q^{3}}{4 p}\left(\log \frac{1}{q}\right) \cos \theta+\frac{q^{3}}{p}\left(\pi p D_{1} G_{3}(p, 0, p, 0)+\frac{1}{16}\right) \cos \theta\right]+O\left(f^{5}(\gamma)\right) .
$$

After applying $\Pi$, the $\cos \theta$ terms vanish and

$$
\|\Pi \mathcal{S}(\psi)\|_{L^{2}}=O\left(f^{5}(\gamma)\right) .
$$

From Lemma 4.1 and 3.22 we deduce that

$$
\left\|\left(\Pi \mathcal{S}^{\prime}(\psi)\right)^{-1}\right\| \leqslant C f(\gamma)
$$

where $\|\cdot\|$ stands for the norm of an operator. Hence

$$
\left\|\left(\Pi \mathcal{S}^{\prime}(\psi)\right)^{-1} \Pi \mathcal{S}(\psi)\right\|_{H^{2}} \leqslant C_{1} f^{6}(\gamma)
$$

Lemma 4.2 and 3.22) imply that

$$
\left\|\mathcal{S}^{\prime \prime}(v)\left(\phi_{1}, \phi_{2}\right)\right\|_{L^{2}} \leqslant C f^{-1}(\gamma)\left\|\phi_{1}\right\|_{H^{2}}\left\|\phi_{2}\right\|_{H^{2}} .
$$

Therefore

and consequently

$$
\|\mathcal{R}(\phi)\|_{L^{2}} \leqslant C f^{-1}(\gamma)\|\phi\|_{H^{2}}^{2}
$$

$$
\left\|\left(\Pi \mathcal{S}^{\prime}(\psi)\right)^{-1} \Pi \mathcal{R}(\phi)\right\|_{H^{2}} \leqslant C_{2}\|\phi\|_{H^{2}}^{2}
$$

Using (5.5), 5.11), 5.6, and 5.13) we find that

$$
\|\mathcal{T}(\phi)\|_{H^{2}} \leqslant C_{1} f^{6}(\gamma)+C_{2} M^{2} f^{12}(\gamma) \leqslant M f^{6}(\gamma)
$$

if $M=2 C_{1}$ and $\gamma$ is sufficiently large. Therefore $\mathcal{T}$ is a map from $D(\mathcal{T})$ into itself.

Next we show that $\mathcal{T}$ is a contraction. Let $\phi_{1}, \phi_{2} \in D(\mathcal{T})$. Note that

$$
\mathcal{T}\left(\phi_{1}\right)-\mathcal{T}\left(\phi_{2}\right)=\left(\Pi \mathcal{S}^{\prime}(\psi)\right)^{-1}(-\Pi)\left(\mathcal{R}\left(\phi_{1}\right)-\mathcal{R}\left(\phi_{2}\right)\right) .
$$

Because

$$
\mathcal{R}\left(\phi_{1}\right)-\mathcal{R}\left(\phi_{2}\right)=\mathcal{S}\left(\psi+\phi_{1}\right)-\mathcal{S}\left(\psi+\phi_{2}\right)-\mathcal{S}^{\prime}(\psi)\left(\phi_{1}-\phi_{2}\right),
$$

we deduce, with the help of 5.12, and 5.6, that

$$
\begin{aligned}
\left\|\mathcal{R}\left(\phi_{1}\right)-\mathcal{R}\left(\phi_{2}\right)\right\|_{L^{2}} & \leqslant\left\|\mathcal{S}^{\prime}\left(\psi+\phi_{2}\right)\left(\phi_{1}-\phi_{2}\right)-\mathcal{S}^{\prime}(\psi)\left(\phi_{1}-\phi_{2}\right)\right\|_{L^{2}}+C f^{-1}(\gamma)\left\|\phi_{1}-\phi_{2}\right\|_{H^{2}}^{2} \\
& \leqslant C f^{-1}(\gamma)\left\|\phi_{2}\right\|_{H^{2}}\left\|\phi_{1}-\phi_{2}\right\|_{H^{2}}+C f^{-1}(\gamma)\left\|\phi_{1}-\phi_{2}\right\|_{H^{2}}^{2} \\
& \leqslant C f^{-1}(\gamma)\left(\left\|\phi_{1}\right\|_{H^{2}}+\left\|\phi_{2}\right\|_{H^{2}}\right)\left\|\phi_{1}-\phi_{2}\right\|_{H^{2}} \leqslant C M f^{5}(\gamma)\left\|\phi_{1}-\phi_{2}\right\|_{H^{2}} .
\end{aligned}
$$

Then 5.10 implies that

$$
\left\|\mathcal{T}\left(\phi_{1}\right)-\mathcal{T}\left(\phi_{2}\right)\right\|_{H^{2}} \leqslant C M f^{6}(\gamma)\left\|\phi_{1}-\phi_{2}\right\|_{H^{2}} .
$$

Therefore $\mathcal{T}$ is a contraction mapping from $D(\mathcal{T})$ to itself when $\gamma$ is sufficiently large. There is a unique fixed point in $D(\mathcal{T})$, which we denote by $\varphi$. 


\section{Minimization}

In this section we prove Theorem 1.1. From now on we emphasize dependencies on $p$. The approximate solution $\psi=\psi(\theta)$ is now denoted by $\psi=\psi(\theta, p)$. By Lemma 5.1 for every $p \in$ $\left[\frac{1}{8 \pi^{2} f^{2}(\gamma)}, \frac{2}{\pi^{2} f^{2}(\gamma)}\right]$ there exists $\varphi=\varphi(\theta, p) \in \mathcal{X}_{*}^{\prime}$ such that $\Pi \mathcal{S}(\psi(\cdot, p)+\varphi(\cdot, p))=0$, i.e. 5.1$\}$ holds. In this section we find a particular $p$ denoted by $p_{\gamma}$ such that $\mathcal{S}\left(\psi\left(\cdot, p_{\gamma}\right)+\varphi\left(\cdot, p_{\gamma}\right)\right)=0$.

Let us denote the set specified by the volume variable $\psi(\cdot, p)+\varphi(\cdot, p)$ by $E_{\varphi(\cdot, p)}$. This notation is consistent with our earlier notation $E_{0}$, the set characterized by $\psi(\cdot, p)$.

LEMMA $6.1 \mathcal{J}\left(E_{\varphi(\cdot, p)}\right)=\mathcal{J}\left(E_{0}\right)+O\left(f^{11}(\gamma)\right)$.

Proof. Expanding $\mathcal{J}\left(E_{\varphi}\right)$ yields

$$
\mathcal{J}\left(E_{\varphi}\right)=\mathcal{J}\left(E_{0}\right)+2 \pi \int_{0}^{2 \pi} \mathcal{S}(\psi) \varphi \mathrm{d} \theta+\frac{2 \pi}{2} \int_{0}^{2 \pi} \mathcal{S}^{\prime}(\psi)(\varphi) \varphi \mathrm{d} \theta+O\left(f^{17}(\gamma)\right) .
$$

The error term in 6.1 is obtained by Lemma 4.2 and the fact $\|\varphi\|_{H^{2}}=O\left(f^{6}(\gamma)\right)$.

On the other hand $\Pi \mathcal{S}(\psi+\varphi)=0$ implies that

$$
\Pi\left(\mathcal{S}(\psi)+\mathcal{S}^{\prime}(\psi)(\varphi)+\mathcal{R}(\varphi)\right)=0
$$

where $\mathcal{R}$ is given in 5.3 . Multiply the last equation by $\varphi$ and integrate to derive, again with the help of Lemma 4.2 .

$$
\int_{0}^{2 \pi} \mathcal{S}(\psi) \varphi \mathrm{d} \theta+\int_{0}^{2 \pi} \mathcal{S}^{\prime}(\psi)(\varphi) \varphi \mathrm{d} \theta=O\left(f^{17}(\gamma)\right)
$$

Consequently, 6.1 becomes

$$
\mathcal{J}\left(E_{\varphi}\right)=\mathcal{J}\left(E_{0}\right)+\frac{2 \pi}{2} \int_{0}^{2 \pi} \mathcal{S}(\psi) \varphi \mathrm{d} \theta+O\left(f^{17}(\gamma)\right) .
$$

Note that 5.9, which follows from Lemma 3.2, and the facts $\varphi \perp \cos \theta$ and $\|\varphi\|_{H^{2}}=O\left(f^{6}(\gamma)\right)$ imply that

$$
\int_{0}^{2 \pi} \mathcal{S}(\psi) \varphi \mathrm{d} \theta=O\left(f^{11}(\gamma)\right)
$$

Therefore

$$
\mathcal{J}\left(E_{\varphi}\right)=\mathcal{J}\left(E_{0}\right)+O\left(f^{11}(\gamma)\right)
$$

This proves the lemma.

Lemma 3.3 shows that

$\mathcal{J}\left(E_{0}\right)=2 \pi^{2} p q+\frac{\gamma}{2}\left(\pi^{2} p q^{4} \log \frac{1}{q}+\frac{\pi^{2} p q^{4}}{4}+2 \pi^{3} p q^{4} G_{1}(p, 0, p, 0)\right)+O\left(\frac{\gamma q^{4}}{p^{2}} \log \frac{p}{q}\right)$.

Sometimes it is convenient to consider rescaled versions of $p$ and $q$. Introduce $P$ and $Q$ so that

$$
p=\frac{P}{2 \pi^{2} f^{2}(\gamma)}, \quad q=f(\gamma) Q, \quad j=1,2 .
$$


By 3.22, and 3.21,

$$
P \in[1 / 4,4], \quad Q \in[1 / 2,2] .
$$

The constraint $2 \pi^{2} p q^{2}=1$ implies that

$$
P Q^{2}=1
$$

In terms of $P$ and $Q, \mathcal{J}\left(E_{0}\right)$ is given by

\section{LEMMA 6.2}

$$
\begin{aligned}
\mathcal{J}\left(E_{0}\right)= & \frac{1}{f(\gamma)}\left(P Q+\frac{P Q^{4}}{2}\right)+\gamma f^{2}(\gamma)\left(\frac{P Q^{4}}{4} \log \frac{1}{Q}+\frac{P Q^{4}}{16}+\frac{\pi P Q^{4}}{2} G_{1}(P, 0, P, 0)\right) \\
& +O\left(f^{5}(\gamma)\right) .
\end{aligned}
$$

Proof. Use 6.2, 3.21, 3.22, and the formula

$$
G_{1}(\lambda r, \lambda z, \lambda s, \lambda t)=G_{1}(r, z, s, t)-\frac{1}{2 \pi} \log \frac{1}{\lambda},
$$

which follows from Lemmas 2.2 (i) and 2.1.

Lemma 6.3 There exists $p_{\gamma} \in\left[\frac{1}{8 \pi^{2} f^{2}(\gamma)}, \frac{2}{\pi^{2} f^{2}(\gamma)}\right]$ such that when $p=p_{\gamma}, \mathcal{S}\left(\varphi\left(\cdot, p_{\gamma}\right)\right)=0$. Moreover

$$
\lim _{\gamma \rightarrow \infty} 2 \pi^{2} f^{2}(\gamma) p_{\gamma}=1 \quad \text { and } \lim _{\gamma \rightarrow \infty} \frac{q_{\gamma}}{f(\gamma)}=1 .
$$

Proof. By Lemmas 6.2, 6.1, and the constraint 6.3,

$$
\begin{aligned}
\mathcal{J}\left(E_{\varphi}\right)= & \frac{1}{f(\gamma)}\left(P Q+\frac{P Q^{4}}{2}\right)+\gamma f^{2}(\gamma)\left(\frac{P Q^{4}}{4} \log \frac{1}{Q}+\frac{P Q^{4}}{16}+\frac{\pi P Q^{4}}{2} G_{1}(P, 0, P, 0)\right) \\
& +O\left(f^{5}(\gamma)\right) \\
= & \frac{1}{f(\gamma)}\left(P Q+\frac{P Q^{4}}{2}\right)+O\left(\gamma f^{2}(\gamma)\right) \\
= & \frac{1}{f(\gamma)}\left(\sqrt{P}+\frac{1}{2 P}\right)+O\left(\gamma f^{2}(\gamma)\right) .
\end{aligned}
$$

We view $\mathcal{J}\left(E_{\varphi(\cdot, p)}\right)$ as a function of $p$, or $P$. Let $P_{\gamma} \in[1 / 4,4]$ be such that at $P=P_{\gamma}, \mathcal{J}\left(E_{\varphi(\cdot, p)}\right)$ is minimized. Since $1 / f(\gamma) \gg \gamma f^{2}(\gamma)$ and $\sqrt{P}+1 /(2 P)$ is minimized at $P=1$, we conclude that $\lim _{\gamma \rightarrow \infty} P_{\gamma}=1$. Let

$$
p_{\gamma}=\frac{P_{\gamma}}{2 \pi^{2} f^{2}(\gamma)}, \quad q_{\gamma}=\sqrt{\frac{1}{2 \pi^{2} p_{\gamma}}} .
$$

As a function of $p, \mathcal{J}\left(E_{\varphi(\cdot, p)}\right)$ is minimized at $p=p_{\gamma}$ and

$$
\lim _{\gamma \rightarrow \infty} 2 \pi^{2} f^{2}(\gamma) p_{\gamma}=1, \quad \lim _{\gamma \rightarrow \infty} \frac{q_{\gamma}}{f(\gamma)}=1 .
$$


It follows from 6.5 that when $\gamma$ is large, $p_{\gamma}$ is in $\left(\frac{1}{8 \pi^{2} f^{2}(\gamma)}, \frac{2}{\pi^{2} f^{2}(\gamma)}\right)$. Therefore

$$
\left.\frac{\mathrm{d} \mathcal{J}\left(E_{\varphi(\cdot, p)}\right)}{\mathrm{d} p}\right|_{p=p_{\gamma}}=0 .
$$

By $(6.6)$ and the invariance of the problem in the $z$-direction, $\mathcal{S}\left(\varphi\left(\cdot, p_{\gamma}\right)\right)=0$. The proof of the last assertion, which we omit, is similar to that of [33, Lemma 4.4].

We have found the solution $E_{\varphi\left(\cdot, p_{\gamma}\right)}$ and completed the proof of Theorem 1.1. The fact that our solution set $E_{\varphi\left(\cdot, p_{\gamma}\right)}$ is close to the region $E_{0}$ bounded by an exact torus comes from the estimate $\|\varphi\|_{H^{2}}=O\left(f^{6}(\gamma)\right)$ in Lemma 5.1 This quantity is much smaller than the size of $\psi(\theta)=p q^{2} / 2+$ $\left(q^{3} \cos \theta\right) / 3$, which is of order 1 .

\section{Discussion}

The stability of the tube solution in $\mathbb{R}^{3}$ is not discussed in this paper. However within the class of axisymmetric sets, the solution we found is stable in some sense. In this class every set is obtained by rotating a two-dimensional subset of $\mathbb{R}_{+}^{2}$ about the $z$-axis, and the stability is reduced to the stability of a 2-dimensional problem in the function space $\mathcal{X}$.

In Section 5 we constructed a family of sets $E_{\varphi(\cdot, p)} \subset \mathbb{R}_{+}^{2}$ identified by the volume variables $\psi(\cdot, p)+\varphi(\cdot, p)$ that satisfy $\Pi \mathcal{S}(\psi(\cdot, p)+\varphi(\cdot, p))=0$. Now we shift each $E_{\varphi(\cdot, p)}$ in $z$-direction by an amount $z$ to a new set $E(p, z)$. As $(p, z)$ varies, $E(p, z)$ forms a two-dimensional manifold

$$
\mathcal{E}=\left\{E(p, z): p \in\left(\frac{1}{8 \pi^{2} f^{2}(\gamma)}, \frac{2}{\pi^{2} f^{2}(\gamma)}\right), z \in \mathbb{R}\right\} .
$$

At each $E(p, 0)$, the space of sets represented by functions in $\mathcal{X}_{*}^{\prime}+\varphi(\cdot, p)$ is transversal to the manifold and has codimension 2 . We denote this space by $\mathcal{F}(p, 0)$, viewed as a fiber space at $E(p, 0)$. Shift each $\mathcal{F}(p, 0)$ in the $z$-direction by an amount $z$ and denote the resulting fiber space at $E(p, z)$ by $\mathcal{F}(p, z)$.

By Lemma 4.1 (ii), Lemma 4.2 and the fact $\Pi \mathcal{S}(\psi(\cdot, p)+\varphi(\cdot, p))=0$, one deduces that $E(p, z)$ is a nondegenerate local minimum of $\mathcal{J}$ in $\mathcal{F}(p, z)$. According to Section $6, E\left(p_{\gamma}, 0\right)=E_{\varphi\left(\cdot, p_{\gamma}\right)}$ is a minimum of $\mathcal{J}$ in the 2 -dimensional manifold $\mathcal{E}$. Hence as a result of the successive minimizations $E\left(p_{\gamma}, 0\right)=E_{\varphi\left(\cdot, p_{\gamma}\right)}$ is a local minimum of $\mathcal{J}$, and hence is stable in the class of axisymmetric sets. Note that since $\mathcal{J}\left(E\left(p_{\gamma}, z\right)\right)=\mathcal{J}\left(E\left(p_{\gamma}, 0\right)\right)$ for all $z \in \mathbb{R}, E\left(p_{\gamma}, 0\right)=E_{\varphi\left(\cdot, p_{\gamma}\right)}$ is a degenerate local minimum of $\mathcal{J}$ due to the translation invariance in the $z$-direction.

In the introduction we mentioned the unit disc solution to 1.1 in $\mathbb{R}^{2}$ and its connection to 1.3 . There are a few other solutions.

In $\mathbb{R}^{2}$ there exists a ring shaped solution of the form $\left\{x \in \mathbb{R}^{2}: 0<R_{1}<|x|<R_{2}\right\}$, if $\gamma$ is greater than a threshold value $\gamma_{0}$; see Kang and Ren [14]. The inner and outer radii $R_{1}$ and $R_{2}$ of the ring solution depend on $\gamma$. There is another threshold $\gamma_{1}>\gamma_{0}$ such that the ring solution is unstable if $\gamma \in\left(\gamma_{0}, \gamma_{1}\right)$ and stable if $\gamma>\gamma_{1}$. This ring solution to 1.1 may be used to construct a solution to (1.3) with multiple, small rings, or to construct a solution to 1.3 with a mix of small rings and small discs [15].

When $\gamma$ is slightly greater than 12 , there is another solution to $\sqrt{1.1}$ in $\mathbb{R}^{2}$ of an oval shape. Corresponding to this oval solution, there exist two solutions to 1.3 , each of which is a small set of an oval shape, as long as the domain $D$ does not possess certain symmetry [35]. The two small 
oval sets center at the same point, but align along two orthogonal directions. The two directions are determined by the shape of $D$.

In $\mathbb{R}^{3}$ the unit ball is a solution to 1.1 . On any bounded domain there exist solutions with multiple components each of which is close to a small ball [34]. The small balls form a body centered cubic pattern, consistent with the spherical morphological phase of diblock copolymers.

There is also a shell shaped solution of the form $\left\{x \in \mathbb{R}^{3}: 0<R_{1}<|x|<R_{2}\right\}$ if $\gamma$ is large enough [28]. However this solution is always unstable.

The toroidal solution found in this paper is a neighborhood around the circle

$$
x^{2}+y^{2}=p^{2}, \quad z=0, \quad(x, y, z) \in \mathbb{R}^{3} .
$$

enclosed by a tube like surface. An interesting point is that the circle is not a geodesic and does not have zero curvature. Also the boundary of our solution is not a constant mean curvature surface. This is in contrast to a result of Mazzeo and Pacard [17]. They showed that if $(M, g)$ is a Riemannian manifold and $\Gamma$ is a closed, nondegenerate geodesic in $M$, then most geodesic tubes of sufficiently small radii around $\Gamma$ can be perturbed into constant mean curvature surfaces. Some kind of converse is also true. If a small tube around any curve $\Gamma$ is a constant mean curvature surface with the second fundamental form bounded then the curve must be a geodesic. This result differs from ours in two aspects: first they need a metric and their curve is a geodesic. Second they do not have the Newtonian potential term. In our case, we constructed a tube around a circle (which is not a geodesic under the Euclidean metric) with the help of the Newtonian potential. Our tube does not have constant mean curvature, but satisfies 1.1 . It is unclear in our case if the circle 7.2 can be replaced by any other curve in $\mathbb{R}^{3}$.

\section{A. Appendix}

The Ohta-Kawasaki free energy functional for a diblock copolymer is a Landau type integral with a unique long range interaction term. The density field of $A$-monomers is given by a function $u$ on a bounded domain $D \subset \mathbb{R}^{n}(n=1,2$, or 3$)$ and the density of $B$-monomers is given by $1-u$. The free energy of a diblock copolymer is

$$
\mathcal{I}_{D}(u)=\int_{D}\left[\frac{\epsilon^{2}}{2}|\nabla u|^{2}+W(u)+\frac{\epsilon \gamma}{2}\left|(-\Delta)^{-1 / 2}(u-a)\right|^{2}\right] \mathrm{d} x
$$

with three parameters $\epsilon>0, \gamma>0$, and $a \in(0,1)$, where $u$ is in

$$
\left\{u \in H^{1}(D): \frac{1}{|D|} \int_{D} u(x) \mathrm{d} x=a\right\} .
$$

The function $W$ is a balanced double well potential such as $W(u)=\frac{1}{4} u^{2}(1-u)^{2}$. In $\left\langle\bar{A} \cdot 1,(-\Delta)^{-1 / 2}\right.$ is the long range interaction term. It is the square root of the inverse of the operator $-\Delta$. The $-\Delta$ operator acts on functions with the zero Neumann boundary condition.

One can show as in [29] that as $\epsilon \rightarrow 0$, the functional $\epsilon^{-1} \mathcal{I}_{D}$ converges, in the sense of the $\Gamma$-convergence theory [6, 21, 20, 16], to a limiting functional $\mathcal{J}_{D}$. The functional $\mathcal{J}_{D}$ is defined on sets whose Lebesgue measure is $a$ times the Lebesgue measure of $D$ and takes the form

$$
\mathcal{J}_{D}(E)=\frac{1}{n-1} \mathcal{P}_{D}(E)+\frac{\gamma}{2} \int_{D}\left|(-\Delta)^{-1 / 2}\left(\chi_{E}-a\right)\right|^{2} \mathrm{~d} x
$$


Here $\mathcal{P}_{D}(E)$ stands for the size of the part of $\partial E$ which is inside $D$, and $\chi_{E}$ is the characteristic function of $E$. The Euler-Lagrange equation of $\mathcal{J}_{D}$ is exactly (1.3).

We mentioned in the introduction that the problem (1.3) is also connected to the GiererMeinhardt system in developmental morphogenesis. The equilibrium state of the system is

$$
\epsilon^{2} \Delta u-u+\frac{u^{p}}{\left(1+\kappa u^{p}\right) v^{q}}=0 \text { in } D ; d \Delta v-v+\frac{u^{r}}{v^{s}}=0 \text { in } D ;\left.\quad \frac{\partial u}{\partial v}\right|_{\partial D}=0 ;\left.\quad \frac{\partial v}{\partial v}\right|_{\partial D}=0,
$$

where $p, q, r$, and $s$ are positive parameters and they satisfy $0<(p-1) / q<r /(s+1)$. Here the constant $\kappa$ is the saturation parameter. We consider the case $\kappa>0$. Then (A.2) is known as the GM system with saturation. We derive a formal singular limit of (A.2) as $\epsilon \rightarrow 0$. It seems possible that one may carry out a rigorous deduction using techniques developed by del Pino, Kowalczyk and Wei [9, 8].

The diffusion coefficient $\epsilon^{2}$ of the variable $u$ must be small. The diffusion coefficient of $v$ must be large in the sense that $d=d_{0} / \epsilon$ where $d_{0}>0$ is independent of $\epsilon$. This is the parameter range considered in [36].

The nonlinearity in the first equation of A.2) is denoted by

$$
f(u, v)=-u+\frac{u^{p}}{\left(1+\kappa u^{p}\right) v^{q}} .
$$

It has a bistable shape with respect to $u$. For each $v>0$, there exist three zeros of $f(u, v)$ as a function of $u$. There is a particular value $v_{0}$ such that at $v=v_{0}, f\left(\cdot, v_{0}\right)$ becomes a balanced cubic nonlinearity, in the sense $\int_{0}^{z} f\left(u, v_{0}\right) \mathrm{d} u=0$. Here $z$ is the largest zero of $f\left(\cdot, v_{0}\right)$.

A subset $E$ of $D$ is expected so that $u(x)$ of a solution $(u, v)$ to (A.2) is close to $z$ if $x$ is in $E$ and close to 0 if $x$ is in $D \backslash E$. The boundary of $E$ in $D$ is a collection of surfaces which we denote by $\Gamma$. The value of $u$ changes abruptly across $\Gamma$. Away from $\Gamma$ we take $u \approx z \chi_{E}$ where $\chi_{E}$ is the characteristic function of $E$. The shape of $u$ near $\Gamma$ is more complicated. Let $Q(\xi, s)$ be the traveling wave solution of the problem

$$
Q_{\xi \xi}+c(s) Q_{\xi}+f(Q, s)=0, \quad \xi \in \mathbb{R} .
$$

In (A.3), $s$ is a parameter. As $\xi$ tends to $-\infty$, we require that $Q(\xi, s)$ tend to 0 , and as $\xi$ tends to $\infty$, we require that $Q(\xi, s)$ tend to the largest zero of $f(\cdot, s)$. The constant $c(s)$ is the velocity of the traveling wave. It is unknown and must be determined from the equation (A.3.

Let $d(x)$ be the signed distance function from a point $x$ to $\Gamma$. The sign of $d(x)$ is positive if $x$ is in $E$ and negative if $x$ is in $D \backslash E$. We assume that approximately

$$
u(x) \approx Q(d(x) / \epsilon, s)
$$

where $s$ is constant, discussed later. Insert this $Q$ into the first equation in (A.2) to find

$$
Q_{\xi \xi}|\nabla d|^{2}+\epsilon Q_{\xi} \Delta d+f(Q, s)=0 .
$$

It is known that on the set $\Gamma,|\nabla d|=1$ and $\Delta d(x)=\mathcal{H}(x)$ where $\mathcal{H}(x)$ is the mean curvature of $\Gamma$ at $x$, viewed from $E$. Therefore we obtain

$$
Q_{\xi \xi}+\epsilon \mathcal{H} Q_{\xi}+f(Q, s)=0 .
$$


Comparing A.4 with $\mathrm{A.3}$ we deduce

$$
c(s)=\epsilon \mathcal{H} .
$$

Now we discuss $c(s)$. On the boundary $\Gamma, s$ must be equal to $v(x)$. However unlike $u(x)$, $v(x)$ changes slowly in $x$. Asymptotically we have the expansion $v(x) \approx v_{0}+\epsilon w(x)$, so that $c(s)=c(v) \approx c\left(v_{0}\right)+\epsilon c^{\prime}\left(v_{0}\right) w$. Since $v_{0}$ is the point where $f\left(\cdot, v_{0}\right)$ is balanced, $c\left(v_{0}\right)=0$. Hence A.5 implies that

$$
\mathcal{H}=c^{\prime}\left(v_{0}\right) w .
$$

It remains to find an equation for $w$. In the second equation of A.2 we deduce

$$
\frac{d_{0}}{\epsilon} \Delta\left(v_{0}+\epsilon w\right)-\left(v_{0}+\epsilon w\right)+\frac{u^{r}}{\left(v_{0}+\epsilon w\right)^{s}}=0 .
$$

As $\epsilon \rightarrow 0$, we find

$$
d_{0} \Delta w-v_{0}+\frac{z^{r}}{v_{0}^{s}} \chi_{E}=0
$$

The equations A.6 and A.7 form a system for the boundary $\Gamma$ :

$$
\mathcal{H}=c^{\prime}\left(v_{0}\right) w \quad \text { on } \Gamma, \quad d_{0} \Delta w-v_{0}+\frac{z^{r}}{v_{0}^{s}} \chi_{E}=0 \quad \text { in } D .
$$

Note that the Neumann boundary condition for $v$ implies the same boundary condition for $w$ and hence

$$
\int_{D}\left(-v_{0}+\frac{z^{r}}{v_{0}^{s}} \chi_{E}\right) \mathrm{d} x=0
$$

Therefore $|E|=\frac{v_{0}^{s+1}}{z^{r}}|D|$. Define

$$
a=\frac{v_{0}^{s+1}}{z^{r}}, \quad \gamma=-\frac{c^{\prime}\left(v_{0}\right) z^{r}}{d_{0} v_{0}^{s}}
$$

to turn $\mathrm{A} .8$ into 1.3 .

\section{Acknowledgments}

Research of X. Ren was supported in part by NSF grant DMS-0907777. Research of J. Wei was supported in part by an earmarked grant of RGC of Hong Kong and Focused Research Scheme of CUHK.

\section{REFERENCES}

1. Alberti, G., Choksi, R., \& OTto, F. Uniform energy distribution for an isoperimetric problem with long-range interactions. J. Amer. Math. Soc. 22 (2009), 569-605. MR 2476783

2. Anderson, B. P., Haljan, P. C., Regal, C. A., Feder, D. L., Collins, L. A., Clark, C. W., \& Cornell, E. A. Watching dark solitons decay into vortex rings in a Bose-Einstein condensate. Phys. Rev. Lett. 86 (2001), 2926-2929. 
3. Batchelor, G. K. An Introduction to Fluid Dynamics. Cambridge Univ. Press (1967). Zbl 0152.44402 MR 1744638

4. Bates, F. S., \& Fredrickson, G. H. Block copolymers-designer soft materials. Phys. Today 52 (1999), 32-38.

5. CHOKSi, R., \& STERnBerg, P. On the first and second variations of a nonlocal isoperimetric problem. J. Reine Angew. Math. 611 (2007), 75-108. Zbl 1132.35029 MR 2360604

6. De Giorgi, E. Sulla convergenza di alcune successioni d'integrali del tipo dell'area. Rend. Mat. (6) 8 (1975), 277-294. Zbl 0316.35036 MR 0375037

7. DEL Pino, M. Radially symmetric internal layers in a semilinear elliptic system. Trans. Amer. Math. Soc. 347 (1995), 4807-4837. Zbl 0853.35009 MR 1303116

8. Del Pino, M., KowalczyK, M., \& Wei, J. Concentration on curves for nonlinear Schrödinger equations. Comm. Pure Appl. Math. 60 (2007), 113-146. Zbl 1123.35003 MR 2270164

9. Del Pino, M., KowAlCZYK, M., \& WeI, J. Resonance and interior layers in an inhomogeneous phase transition model. SIAM J. Math. Anal. 38 (2007), 1542-1564. Zbl pre05194945 MR 2286019

10. Fife, P. C., \& Hilhorst, D. The Nishiura-Ohnishi free boundary problem in the 1D case. SIAM J. Math. Anal. 33 (2001), 589-606. Zbl 1077.35121 MR 1871411

11. Ghergu, M., \& Rădulescu, V. Singular Elliptic Problems: Bifurcation and Asymptotic Analysis. Oxford Lecture Ser. Math. Appl. 37, Clarendon Press, Oxford, 2008. Zbl 1159.35030 MR 2488149

12. Ghergu, M., \& RĂdulescu, V. A singular Gierer-Meinhardt system with different source terms. Proc. Roy. Soc. Edinburgh Sect. A 138 (2008), 1215-1234. Zbl 1171.35396 MR 2488056

13. Gierer, A., \& Meinhardt, H. A theory of biological pattern formation. Kybernetik 12 (1972), 30-39.

14. KANG, X., \& REN, X. Ring pattern solutions of a free boundary problem in diblock copolymer morphology. Phys. D 238 (2009), 645-665. Zbl 1160.37386 MR 2590243

15. KANG, X., \& REN, X. The pattern of multiple rings from morphogenesis in development. J. Nonlinear Sci., to appear.

16. Kohn, R., \& Sternberg, P. Local minimisers and singular perturbations. Proc. Roy. Soc. Edinburgh Sect. A 111 (1989), 69-84. Zbl 0676.49011 MR 0985990

17. Mazzeo, R., \& PaCARD, F. Foliations by constant mean curvature tubes. Comm. Anal. Geom. 13 (2005), 633-670. Zbl 1096.53035 MR 2191902

18. Meinhardt, H. Models of Biological Pattern Formation. Academic Press, London (1982).

19. Meinhardt, H. The Algorithmic Beauty of Sea Shells. 2nd ed., Springer, Berlin (1998).

20. ModicA, L. The gradient theory of phase transitions and the minimal interface criterion. Arch. Ration. Mech. Anal. 98 (1987), 123-142. Zbl 0616.76004 MR 0866718

21. Modica, L., \& Mortola, S. Un esempio di $\Gamma^{-}$-convergenza. Boll. Un. Mat. Ital. B (5) 14 (1977), 285-299. Zbl 0356.49008 MR 0445362

22. Murray, J. Mathematical Biology II: Spatial Models and Biomedical Applications. 3rd ed., Springer (2008)

23. NishiURA, Y., \& OHNishi, I. Some mathematical aspects of the microphase separation in diblock copolymers. Phys. D 84 (1995), 31-39. Zbl :1194.82069 MR 1334695

24. ОhtA, T., \& KAWASAKI, K. Equilibrium morphology of block copolymer melts. Macromolecules 19 (1986), 2621-2632.

25. OshitA, Y. Singular limit problem for some elliptic systems. SIAM J. Math. Anal. 38 (2007), 1886-1911. Zbl 1141.35021 MR 2299434

26. Pochan, D. J., Chen, Z., Cui, H., Hales, K., QI, K., \& Wooley, K. L. Toroidal triblock copolymer assemblies. Science 306 (2004), 94-97.

27. Rayfield, G. W., \& Reif, F. Quantized vortex rings in superfluid helium. Phys. Rev. 136 (1964), A1194-A1208. 
28. REN, X. Shell structure as solution to a free boundary problem from block copolymer morphology. Discrete Contin. Dynam. Systems 24 (2009), 979-1003. Zbl :1178.35386 MR 2505690

29. REN, X., \& WEI, J. On the multiplicity of solutions of two nonlocal variational problems. SIAM J. Math. Anal. 31 (2000), 909-924. Zbl 0973.49007 MR 1752422

30. REN, X., \& WEI, J. Concentrically layered energy equilibria of the di-block copolymer problem. Eur. J. Appl. Math. 13 (2002), 479-496. Zbl 1010.82041 MR 1939157

31. REN, X., \& WEI, J. Existence and stability of spherically layered solutions of the diblock copolymer equation. SIAM J. Appl. Math. 66 (2006), 1080-1099. Zbl 1103.34046 MR 2216732

32. REN, X., \& WEI, J. Many droplet pattern in the cylindrical phase of diblock copolymer morphology. Rev. Math. Phys. 19 (2007), 879-921. Zbl 1145.82007 MR 2349026

33. REN, X., \& WEI, J. Single droplet pattern in the cylindrical phase of diblock copolymer morphology. J. Nonlinear Sci. 17 (2007), 471-503. Zbl 1134.82048 MR 2361417

34. REN, X., \& WEI, J. Spherical solutions to a nonlocal free boundary problem from diblock copolymer morphology. SIAM J. Math. Anal. 39 (2008), 1497-1535. Zbl 1153.35091 MR 2377287

35. REN, X., \& WeI, J. Oval shaped droplet solutions in the saturation process of some pattern formation problems. SIAM J. Appl. Math. 70 (2009), 1120-1138. Zbl 1198.35009 MR 2546355

36. SAкамото, K., \& SUZUKi, H. Spherically symmetric internal layers for activator-inhibitor systems I. Existence by a Lyapunov-Schmidt reduction. J. Differential Equations 204 (2004), 56-92. Zbl 1068.35013 MR 2076159

37. Sternberg, P., \& Topaloglu, I. A note on the global minimizers of the nonlocal isoperimetric problem in two dimensions. Interfaces Free Bound. 13 (2011), 155-169. 